Ivan Merdukh*

\title{
The Electromagnetic-Field Effect of Industrial Origin on the Cardio-Vascular System of Urban Land Inhabitants (Illustrated with an Example of Ivano-Frankivsk City, Ukraine)
}

\section{Introduction}

The health level of people is the key indicator of the optimized social ecological system. Modern society creates and makes the best use of innovative technologies in the field of science and technology that allow satisfying its growing needs. Such a type of social development has to provide a dynamic balance in the system "society - natural environment" - in the social ecological system. The most lability in the context of technocratic society is the balance "society - natural environment" in the urban ecological systems (urbanized social ecological systems). The level increase of comfort of the people in the urban social ecosystems has led to becoming a greater number of factors that can make affects on the balance of the system "society - natural environment" and, consequently, on the people health. Among them the special place takes the influence of the electromagnetic radiation (EMR) on the human body.

To control the influence of electromagnetic fields (EMF) of industrial origin on the human body was developed "The State Sanitary Regulations and Protection regulation of the people from an influence of electromagnetic radiation" approved by the order of The Ministry of Healthcare of Ukraine No. 239 dtd. 8.01.1996. This document is the rules and regulations that protect people from an exposure of electromagnetic fields and includes chapter "The Sanitary Regulations and Protection regulations of the people from an influence of electromagnetic fields forming the radio technical objects" and "The Sanitary and Protection Regulations of the people from electric field exposure, creating facilities of power transmission of industrial frequency". In accordance with this document "To the electromagnetic sources in the populated area belong the radio, television and radar stations of various profiles that are at work in the radio frequency band, and transmission network, which consists of air high-voltage power line and electrical substations. To the substations may belong: switchgear, electricity transformers, transformers, rectifier units and other facilities and constructions".

\footnotetext{
* Ivano-Frankivsk Oil and Gas National Technical University, Ivano-Frankivsk, Ukraine
} 
But, according to this document the maximum permissible limt (MPL) of electromagnetic fields that apply to the frequency range from $30 \mathrm{kHz}$ to $300 \mathrm{GHz}$, is much lower as such level in Russia and significantly lower as such level in Europe. In addition, a list of radio technical objects (RTO) that radiate electromagnetic energy into the environment, needs to be extended due to the use of digital radio relay stations and base stations of mobile communication. The Regulation of the Board of Ministers of Ukraine No. $808 \mathrm{dtd}$. 28.08.2013 defines "The list of activities and objects of increased ecological hazard". In the clause 26 of this list are mentioned the radio technical objects, which have increased ecological hazard: radio transmitting stations, radio, TV and radar stations, digital radio relay stations, the base stations of mobile communication.

That is why the regulatory base that governs the maximum permissible limit (MPL) of electromagnetic fields in Ukraine needs to be renewed, and the influence of electromagnetic waves in the radio frequency on the social component of ecological safety of urban social ecological systems has a grate interest of scientific.

\section{Methods of Ecological and Geographical Researches}

For decision of given tasks there was chosen an urban social ecological system of Ivano-Frankivsk city. In urban land of Ivano-Frankivsk city that was chosen as a test area, were defined 122 points at a distance of $200-500 \mathrm{~m}$. The choice of control points of the test area was conducted in accordance with specific of building area including large, crowded places with probably increased electromagnetic fields: educational institutions, health facilities, religious structures, big domestic buildings, bridges over the river Bystrytsa Nadvirnyanska and Bystrytsa Solotvynska, railway station, railway track and bridges across it, big shops, cinemas, bus stops on streets with trolley movement and others.

The urban ecological system in Ivano-Frankivsk city takes an area of $51.36 \mathrm{~km}^{2}$, total area of Ivano-Frankivsk city $-83.73 \mathrm{~km}^{2}$ [12]. The average density of placement of control points on the test area is 2.4 points on $1 \mathrm{~km}^{2}$, and in the central part of the city the density of placement of the control points is slightly higher.

At each point of the test area the intensity of the electric field $(E)$, the intensity of the magnetic field $(H)$ and the intensity of the surface density of energy flow $(\mu)$ were measured. For the measurements tester Tenmars RF three-Axis Field Strength Meter TM-195 were used [13]. The facility is used to measure electromagnetic fields of anthropogenic origin in the frequency range from $50 \mathrm{MHz}$ to $3.5 \mathrm{GHz}$, in particular for measuring the field intensity of high-frequency electromagnetic waves, surface density of energy flow of antennas for mobile communication databases, units for wireless communication (CW, TDMA, GSM, DECT), transmitters, wireless networks LAN (Wi-Fi), the leak detection of microwaves, determination of safety radiation level of mobile phones, the determination of electromagnetic safety of work and domestic establishments. The facility is working with discretization interval - three 
measurements in 1 second and allows making measures of immediate, average and maximum measured value. For isotopic measurements of electromagnetic fields the facility is equipped with three-channel sensor. The settings allow for the use of a separate axis X, Y or Z sensor (non-isotopic measurements of electromagnetic field), or all axis in the same time (isotopic measurements of the electromagnetic field).

At each point of the test area was conducted isotopic measurements of EMF at a distance of $1.8 \mathrm{~m}$ from the soil surface during 10 minutes, and the facility was placed immovably at a distance not less than $1 \mathrm{~m}$ from the experimenter. Also the facility was placed with the sensor towards potential sources of electromagnetic radiation. Received data are presented in the Table 1.

The space around the source of EMF conventionally is divided into the near zone (zone induction) and distant zone (zone of radiation). The near zone covers the space with a radius of about $1 / 6$ wave length. In this zone the electromagnetic wave is not formed yet because the intensity of EMF is measured separately with intension of magnetic and electric field components, negative effect of EMF in this area is mainly caused by the electrical component.

In accordance with the order of the Ministry of Healthcare of Ukraine No. 1040 dtd. 29.11.2013 "About approval of the measurements methodology for levels distribution of electromagnetic fields" including the "The State Sanitary Regulations and Protection Regulations of the people from the influence of electromagnetic radiation", approved by the order of the Ministry of Healthcare of Ukraine No. 239 dtd. 01.08.1996, the maximum permissible limit (MPL) of EMF in the frequency range of $30-300 \mathrm{MHz}$ is $3 \mathrm{~V} / \mathrm{m}$ and in a frequency range from $300 \mathrm{MHz}$ to $300 \mathrm{GHz}-$ $2.5 \mathrm{~mW} / \mathrm{cm}^{2}$. For comparison, in the Russian Federation and in Belarus this rate is $10 \mathrm{mkV} / \mathrm{cm}^{2}$, and in some European countries - $100 \mathrm{~mW} / \mathrm{cm}^{2}$.

The received data show the maximum permissible limit (MPL) of surface density of energy flow of the electromagnetic field in terms of average values of this factor in the such streets as Evgen Konovalets street - Sichovi Striltsi street - Academician Sakharov street - Vyacheslav Chornovil street (Fig. 1), in terms of the maximum values of this factor in the such streets as Evgen Konovalets street - Sichovi Striltsi street - Academician Sakharov street - Vyacheslav Chornovil street; Zaliznychna street - Mariya Pidhiryanka street - Nezaleyhnosti street; Pylyp Orlyk street (Fig. 2).

The elevation of the maximum permissible limit (MPL) of electric field intensity in terms of average values of this factor are seen in Korolya Danyla street - Evgen Konovalets street - Sichovi Striltsi street - Academician Sakharov street - Vyacheslav Chornovil street - Nezalezhnosti street - Pryvoksalna street (Fig. 3), in terms of the maximum values of this factor Korolya Danyla street - Evgen Konovalets street - Sichovi Striltsi street - Academician Sakharov street - Shevchenko street - Vyacheslav Chornovil street - Nezalezhnosti street - Pryvoksalna street and the intersection Ivasyuka - Vovchynetska street and Ivasyuka - Tysmenytska street (Fig. 4). The maps are created using geographic information system MAP INFO, and the points of test area are marked using the service Google Maps. 


\begin{tabular}{|c|c|c|c|c|c|c|c|c|c|c|c|c|c|c|c|c|c|}
\hline \multirow{3}{*}{ 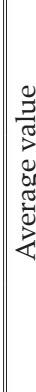 } & 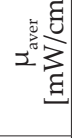 & 仝 & $\begin{array}{l}\stackrel{+}{+} \\
\stackrel{0}{0}\end{array}$ & $\begin{array}{c}\widetilde{1} \\
\text { S. }\end{array}$ & $\begin{array}{l}\stackrel{2}{\infty} \\
\stackrel{\infty}{-} \\
\sim\end{array}$ & $\begin{array}{l}\infty \\
\stackrel{\infty}{0} \\
\stackrel{0}{0}\end{array}$ & $\begin{array}{l}\infty \\
\substack{\infty\\
}\end{array}$ & $\overrightarrow{\tilde{N}}$ & $\begin{array}{l}\hat{\theta} \\
\stackrel{0}{0} \\
0\end{array}$ & $\begin{array}{l}\text { مी } \\
\stackrel{2}{0}\end{array}$ & $\stackrel{m}{0} \stackrel{0}{0}$ & $\begin{array}{l}0 \\
\\
\vdots \\
0\end{array}$ & 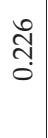 & $\begin{array}{c}1 \\
m \\
0 \\
0\end{array}$ & $\begin{array}{l}\infty \\
\vdots \\
0 \\
0\end{array}$ & $\stackrel{+}{\stackrel{+}{C}}$ & $\begin{array}{l}\stackrel{a}{0} \\
\text { Oे }\end{array}$ \\
\hline & $\beth^{\mathrm{E}}$ & $\begin{array}{l}\stackrel{\sim}{1} \\
\infty \\
\end{array}$ & 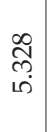 & $\begin{array}{l}\hat{O} \\
\infty \\
\infty\end{array}$ & $\begin{array}{l}02 \\
0 \\
\infty \\
\infty\end{array}$ & 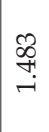 & $\begin{array}{l}\hat{\hat{O}} \\
\text { 0. }\end{array}$ & $\begin{array}{l}\text { ボ } \\
\text { ஸे }\end{array}$ & 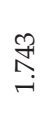 & $\begin{array}{c}\tilde{\sigma} \\
\vec{\sim}\end{array}$ & 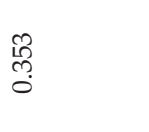 & 拿 & $\begin{array}{l}\underset{T}{\sim} \\
\stackrel{\oplus}{m}\end{array}$ & $\begin{array}{l}\infty \\
\infty \\
\infty \\
-i\end{array}$ & $\begin{array}{l}\infty \\
\infty \\
\infty \\
0 \\
0\end{array}$ & مْ & $\stackrel{\vec{m}}{\vec{m}}$ \\
\hline & W & $\begin{array}{l}\hat{\omega} \\
\text { N̦ } \\
\text { ले }\end{array}$ & 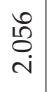 & $\begin{array}{l}\stackrel{\infty}{\hat{~}} \\
\stackrel{i}{i}\end{array}$ & 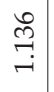 & $\begin{array}{l}\infty \\
\infty \\
10 \\
0\end{array}$ & $\underset{i}{\stackrel{i}{i}}$ & 孚 & $\begin{array}{l}\text { त̂ } \\
\text { ஸै }\end{array}$ & $\begin{array}{l}\stackrel{10}{P} \\
\stackrel{0}{0} \\
0\end{array}$ & کू & $\begin{array}{c}\stackrel{1}{2} \\
\widehat{O} \\
0\end{array}$ & $\begin{array}{l}\vec{I} \\
\infty \\
0 \\
0\end{array}$ & 命 & 尔 & 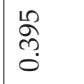 & 占 \\
\hline \multirow{3}{*}{ 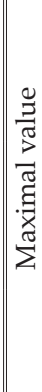 } & 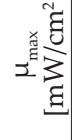 & $\begin{array}{l}\infty \\
\infty \\
\stackrel{\infty}{\circ}\end{array}$ & 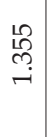 & 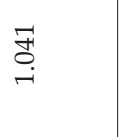 & $\begin{array}{c}\hat{\omega} \\
\infty \\
m \\
m\end{array}$ & İ & ڤ్ & \begin{tabular}{l}
$\mathcal{N}$ \\
\multirow{2}{\infty}{} \\
0
\end{tabular} & 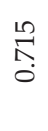 & $\begin{array}{l}3 \\
3 \\
0 \\
0\end{array}$ & 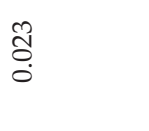 & 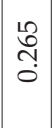 & $\begin{array}{l}\hat{\theta} \\
\stackrel{-}{-}\end{array}$ & 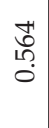 & 全 & 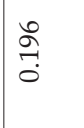 & 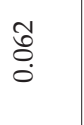 \\
\hline & 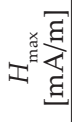 & $\begin{array}{l}\frac{1}{\dddot{P}} \\
\stackrel{9}{\sigma}\end{array}$ & 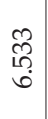 & 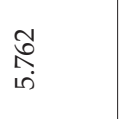 & $\begin{array}{l}\hat{\sigma} \\
\sigma\end{array}$ & م̂ح & & $\begin{array}{l}\infty \\
\stackrel{\infty}{0} \\
+ \\
+\end{array}$ & $\begin{array}{l}\infty \\
\text { \& } \\
\text { மn }\end{array}$ & $\begin{array}{l}\mathscr{2} \\
\stackrel{\sigma}{2} \\
\stackrel{0}{1}\end{array}$ & $\begin{array}{l}8 \\
\infty \\
\infty \\
0\end{array}$ & $\begin{array}{c}\infty \\
m \\
m \\
m\end{array}$ & $\begin{array}{l}\hat{N} \\
\hat{\sigma} \\
\text { ம் }\end{array}$ & $\begin{array}{l}\hat{\omega} \\
\stackrel{n}{n}\end{array}$ & م⿱乛龰 & ถิ & مִ \\
\hline & 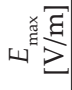 & $\begin{array}{l}l \\
\stackrel{\circ}{\circ} \\
\end{array}$ & 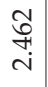 & $\underset{\vec{\lambda}}{\vec{i}}$ & $\begin{array}{l}\infty \\
\stackrel{N}{N} \\
\infty \\
\end{array}$ & $\stackrel{\infty}{\stackrel{\infty}{\hookrightarrow}}$ & $\bar{\sigma}$ & $\begin{array}{l}\hat{N} \\
\text { م̣ } \\
-\end{array}$ & ڤ్ & 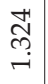 & $\begin{array}{c}\text { ల్ } \\
\text { ֻై }\end{array}$ & 总 & 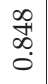 & \begin{tabular}{l}
0 \\
\multirow{0}{0}{} \\
0
\end{tabular} & $\begin{array}{l}\infty \\
\infty \\
i \\
0 \\
0\end{array}$ & 囯 & 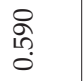 \\
\hline \multirow{3}{*}{ 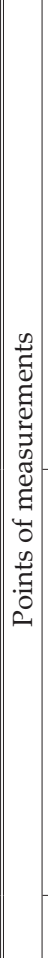 } & 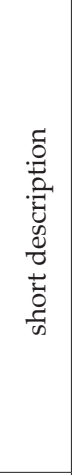 & 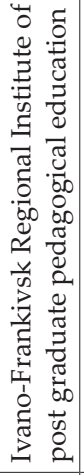 & 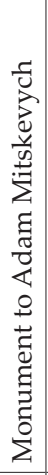 & 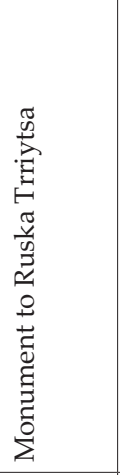 & 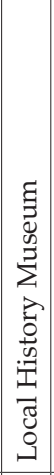 & 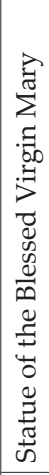 & 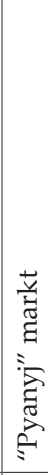 & 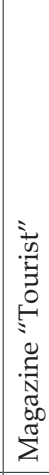 & 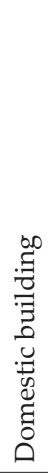 & 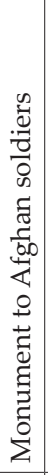 & 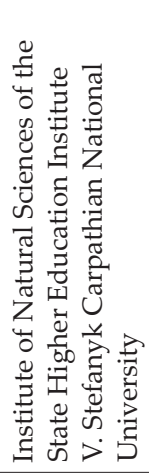 & 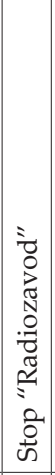 & 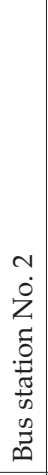 & 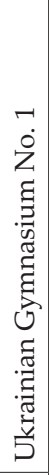 & 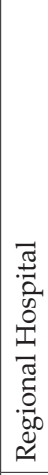 & 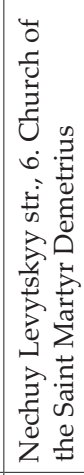 & 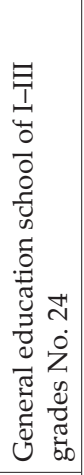 \\
\hline & 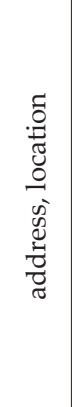 & 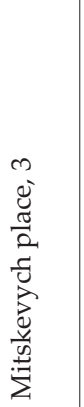 & 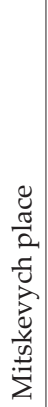 & 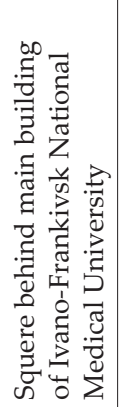 & 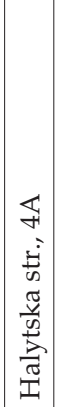 & 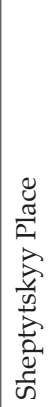 & 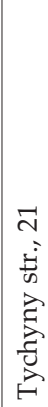 & 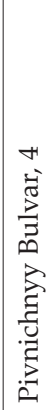 & 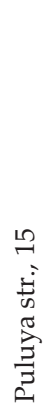 & 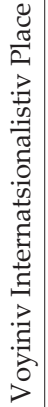 & 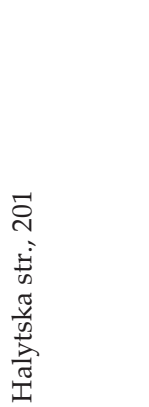 & 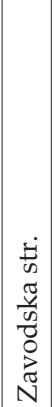 & 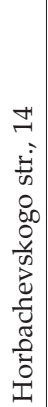 & 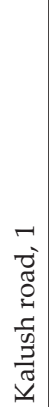 & 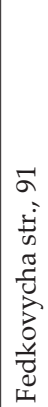 & 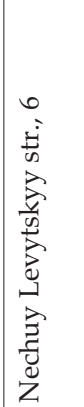 & 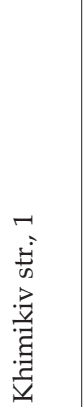 \\
\hline & $\dot{z}$ & - & $N$ & $n$ & $\theta$ & in & 6 & $\wedge$ & $\infty$ & $a$ & $\stackrel{\circ}{=}$ & $\exists$ & $\approx$ & $\stackrel{m}{\longrightarrow}$ & $\ddot{t}$ & $\stackrel{2}{\sim}$ & $\stackrel{0}{\sim}$ \\
\hline
\end{tabular}




\begin{tabular}{|c|c|c|c|c|c|c|c|c|c|c|c|c|c|c|c|c|c|c|}
\hline 0 & Iี & 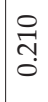 & ठे & 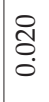 & 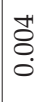 & 왕 & 感 & 苟 & $\mid \begin{array}{c}10 \\
0 \\
0 \\
0\end{array}$ & 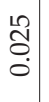 & $\begin{array}{l}\overrightarrow{\gamma ే} \\
\stackrel{0}{0}\end{array}$ & ڤે & 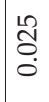 & $\stackrel{\sim}{\Xi}$ & סू & & 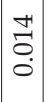 & $\stackrel{2}{0}$ \\
\hline
\end{tabular}

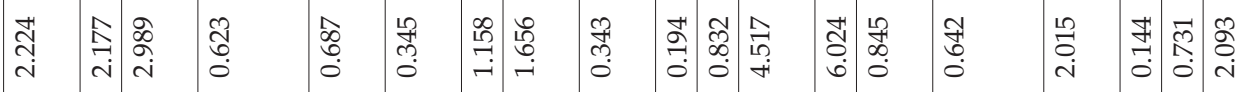

\begin{tabular}{|c|c|c|c|c|c|c|c|c|c|c|c|c|c|c|c|c|c|}
\hline ஜે & ن & స̃ & 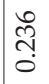 & $\begin{array}{l}+1 \\
0 \\
0 \\
0\end{array}$ & 拿 & 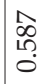 & 孚 & 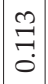 & 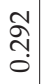 & $\begin{array}{l}\stackrel{L}{\ell} \\
\text { i⿱ } \\
\text { in }\end{array}$ & $\begin{array}{l}\text { ON } \\
\text { N }\end{array}$ & סू & 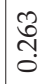 & 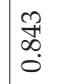 & है & $\begin{array}{c}\tilde{\delta} \\
\tilde{\delta} \\
0\end{array}$ & $\begin{array}{l}\text { F } \\
\stackrel{5}{0}\end{array}$ \\
\hline : & $\begin{array}{l}0 \\
\mathbb{0} \\
\infty \\
0 \\
0\end{array}$ & $\begin{array}{l}\infty \\
\infty \\
\tilde{\delta} \\
0 \\
0\end{array}$ & 苜 & $\begin{array}{ll}10 \\
\vdots \\
0 \\
0\end{array}$ & 莣 & 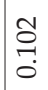 & $\underset{\partial}{\sigma}$ & $\begin{array}{l}1 \\
\delta \\
0 \\
0\end{array}$ & $\underset{\stackrel{g}{+}}{\stackrel{5}{0}}$ & 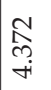 & $\begin{array}{l}\text { f́ } \\
\text { के }\end{array}$ & $\begin{array}{l}\infty \\
\text { ơ } \\
0 \\
0\end{array}$ & $\underset{F}{F}$ & 吕 & $\stackrel{\Delta}{0}$ & 敬 & 尽 \\
\hline
\end{tabular}

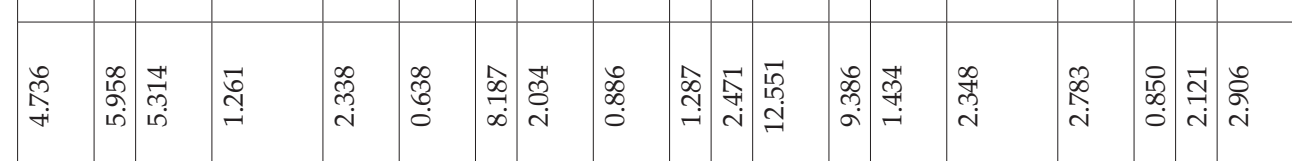

\begin{tabular}{|c|c|c|c|c|c|c|c|c|c|c|c|c|c|c|c|c|c|c|}
\hline 뇸 & 岺 & $\begin{array}{l}\stackrel{\sim}{\sim} \\
\underset{i}{i}\end{array}$ & 导 & $\begin{array}{l}0 \\
\infty \\
\infty \\
0 \\
\dot{0}\end{array}$ & 莽 & $\begin{array}{l}\infty \\
\mathscr{\infty} \\
\ddot{\infty}\end{array}$ & $\begin{array}{l}\stackrel{R}{\hat{N}} \\
0\end{array}$ & $\begin{array}{l}10 \\
\infty \\
\infty \\
0\end{array}$ & $\begin{array}{l}0 \\
1 \\
\text { m. } \\
0\end{array}$ & $\begin{array}{l}\delta \\
\delta \\
\delta\end{array}$ & $\begin{array}{l}\vec{\curvearrowright} \\
\stackrel{\sim}{+}\end{array}$ & $\underset{\hat{N}}{\stackrel{\hat{n}}{2}}$ & $\begin{array}{l}0 \\
0 \\
10 \\
0\end{array}$ & $\begin{array}{l}\infty \\
\infty \\
\infty \\
0\end{array}$ & 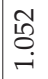 & 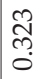 & & $\begin{array}{l}12 \\
\text { o. } \\
\text { - }\end{array}$ \\
\hline
\end{tabular}

\begin{tabular}{|c|c|c|c|c|c|c|c|c|c|c|c|c|c|c|c|c|c|c|}
\hline 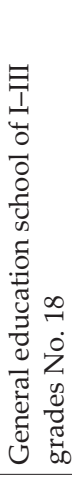 & 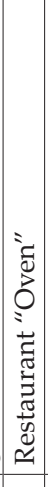 & 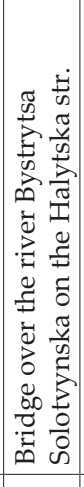 & 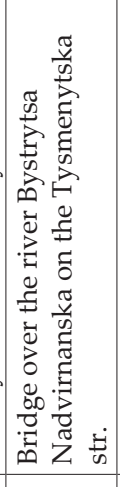 & 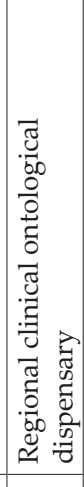 & 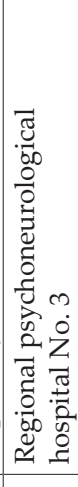 & 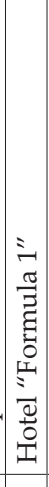 & 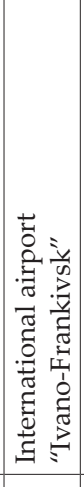 & 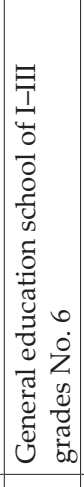 & 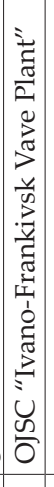 & 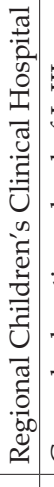 & 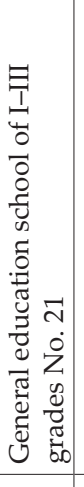 & 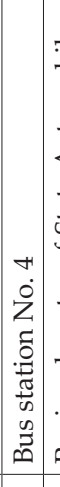 & 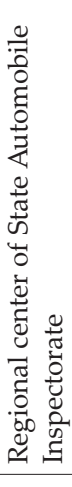 & 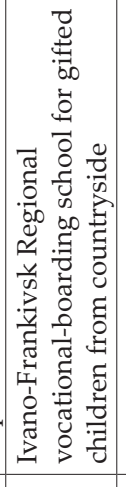 & 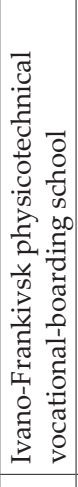 & 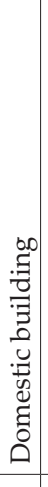 & 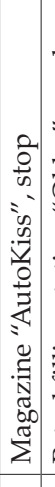 & 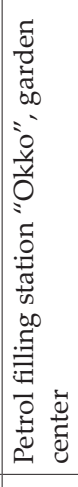 \\
\hline 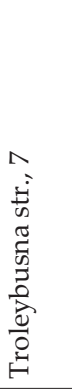 & 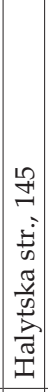 & 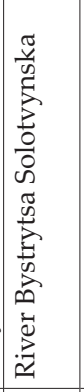 & 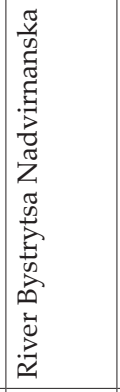 & 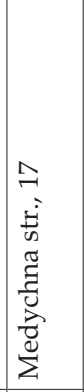 & 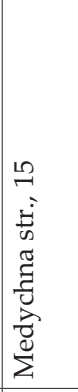 & 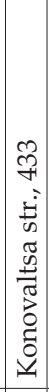 & 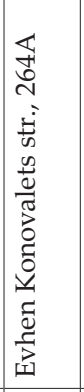 & 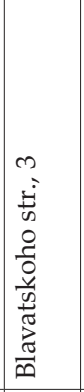 & 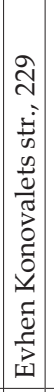 & 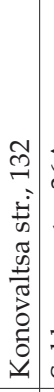 & 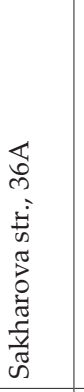 & 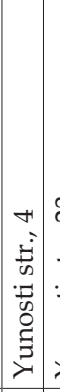 & 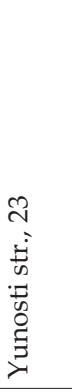 & 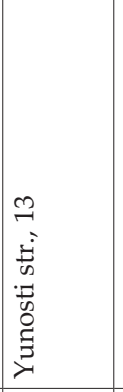 & 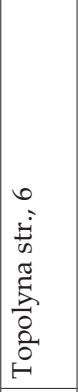 & 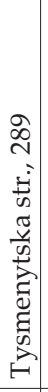 & 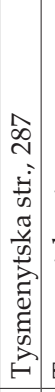 & 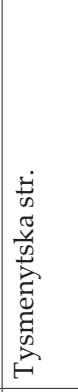 \\
\hline 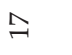 & $\stackrel{\infty}{\sim}$ & $\stackrel{2}{-}$ & ㄱ. & $\vec{N}$ & $\tilde{N}$ & $\tilde{N}$ & A & $\stackrel{\stackrel{\sim}{\sim}}{ }$ & $\stackrel{\sim}{\sim}$ & ลे & $\stackrel{\sim}{\sim}$ & 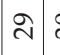 & के & $\bar{m}$ & ๙ิ & $\ddot{m}$ & கొ & $\stackrel{10}{m}$ \\
\hline
\end{tabular}




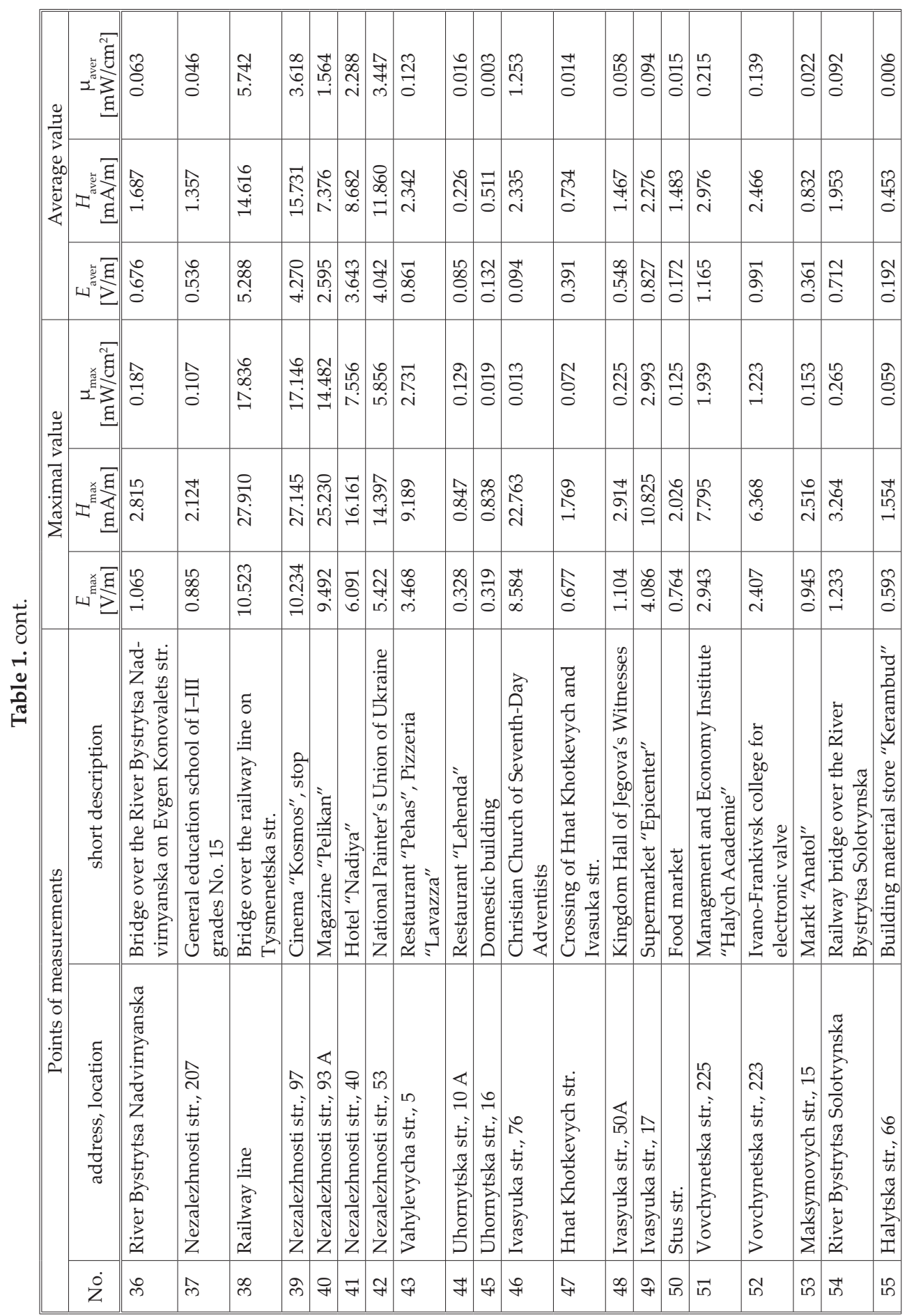




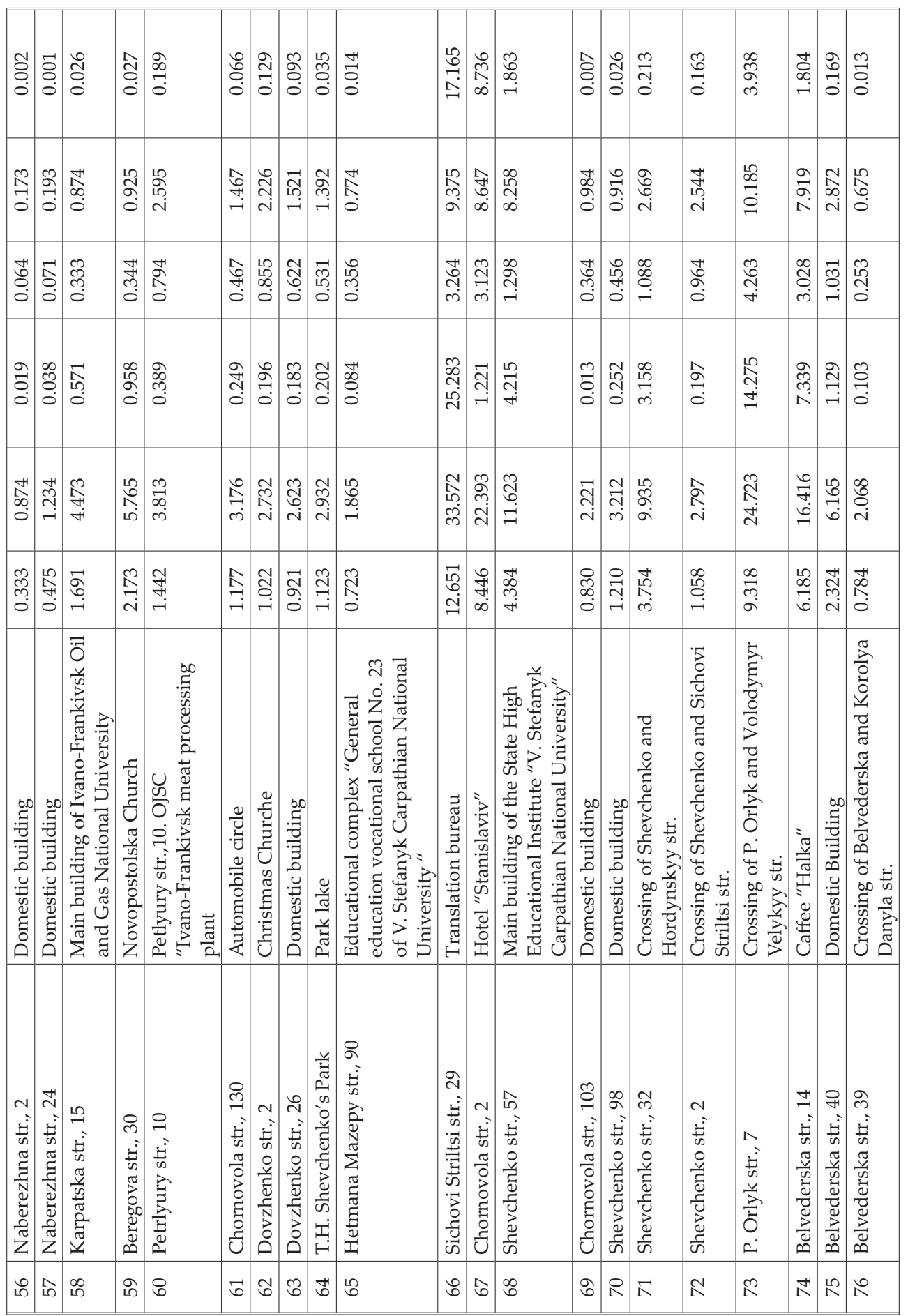




\begin{tabular}{|c|c|c|c|c|c|c|c|c|c|c|c|c|c|c|c|c|c|c|c|c|c|}
\hline \multirow{3}{*}{ 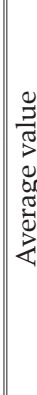 } & $=\frac{i}{\tilde{g}}$ & 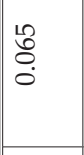 & 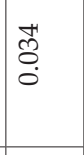 & 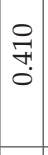 & 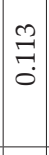 & 站 & $\begin{array}{l}\mathbb{H} \\
0 \\
0\end{array}$ & ठ̊. & 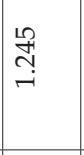 & 盛 & $\mid \begin{array}{l}\infty \\
0 \\
0 \\
0\end{array}$ & $\vec{\sigma}$ & $\begin{array}{l}\text { Oै } \\
0 \\
0 \\
0\end{array}$ & 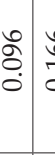 & & 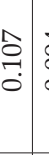 & 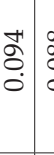 & & $\begin{array}{l}0 \\
0 \\
0 \\
0\end{array}$ & $\begin{array}{l}20 \\
0 \\
0 \\
0\end{array}$ & oे \\
\hline & $I^{2}$ & 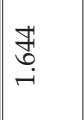 & 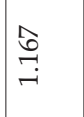 & $\begin{array}{c}\stackrel{L}{N} \\
\mathrm{~N} \\
m\end{array}$ & 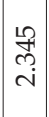 & $\begin{array}{l}3 \\
\stackrel{3}{0} \\
\stackrel{-}{-}\end{array}$ & $\left|\begin{array}{l}2 \\
\infty \\
0 \\
- \\
-\end{array}\right|$ & 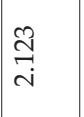 & $\frac{\infty}{\stackrel{\infty}{N}}$ & $\stackrel{\leftrightarrow}{\stackrel{2}{\leftrightarrows}}$ & 点 & $\mid$\begin{tabular}{l|l}
$\infty$ & 0 \\
0 & 0 \\
0 & 1 \\
0 &
\end{tabular} & $\begin{array}{l}\stackrel{+}{D} \\
\stackrel{+}{\rightleftarrows}\end{array}$ & 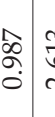 & 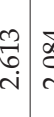 & 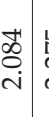 & 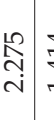 & $\stackrel{+}{\stackrel{+}{r}}$ & 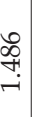 & $\begin{array}{l}0 \\
\sim \\
\text { ஸे } \\
-\end{array}$ & $\begin{array}{l}0 \\
6 \\
0 \\
0\end{array}$ \\
\hline & $H^{*} \bar{z}$ & 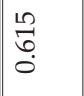 & İ & $\underset{\stackrel{\leftrightarrow}{+}}{\stackrel{\leftrightarrow}{-}}$ & 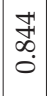 & $\begin{array}{l}\tilde{N} \\
\widetilde{\sigma} \\
0\end{array}$ & \begin{tabular}{|l|}
0 \\
$\stackrel{n}{2}$ \\
0 \\
0
\end{tabular} & 点 & 令 & đ̊. & \begin{tabular}{|l|}
\llcorner \\
$\stackrel{2}{0}$ \\
$\hat{0}$
\end{tabular} & 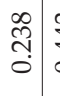 & $\stackrel{\mathscr{B}}{\underset{7}{*}}$ & 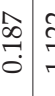 & & 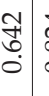 & \begin{tabular}{c|c}
$\mathbb{N}$ & $\delta$ \\
0 & $\vdots$ \\
0 &
\end{tabular} & \begin{tabular}{l|l}
$\infty$ & 1 \\
$\infty$ & 1 \\
0 & \\
0 &
\end{tabular} & 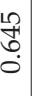 & 命 & 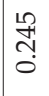 \\
\hline \multirow{3}{*}{ 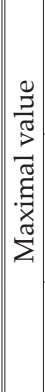 } & $=\frac{\tilde{g}}{3}$ & 苍 & 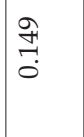 & 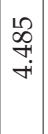 & $\begin{array}{l}\infty \\
\vdots \\
- \\
-i \\
-1\end{array}$ & స్ & $\mid \begin{array}{l}0 \\
\\
0\end{array}$ & $\underset{\widetilde{N}}{\sim}$ & $\begin{array}{l}\infty \\
\tilde{n} \\
i n \\
i n\end{array}$ & సे & $\mid \begin{array}{c}0 \\
\text { ले } \\
\text { o. }\end{array}$ & $\stackrel{0}{0}$ & $\begin{array}{l}\widehat{\widehat{A}} \\
\stackrel{\mathrm{O}}{0}\end{array}$ & 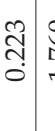 & 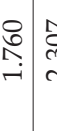 & \begin{tabular}{c|c}
$\hat{c}$ \\
co \\
i
\end{tabular} & 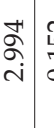 & مै & : & 离 & 总 \\
\hline & $I^{\stackrel{\circ}{E}}$ & $\begin{array}{l}\hat{N} \\
\text { مิ } \\
\text { ம் }\end{array}$ & $\begin{array}{l}20 \\
\stackrel{\infty}{+} \\
\stackrel{i}{+}\end{array}$ & 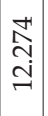 & $\begin{array}{l}\mathbb{Z} \\
\infty \\
\infty \\
1 \\
\qquad\end{array}$ & $\begin{array}{l}\infty \\
\infty \\
\infty \\
\infty \\
\infty\end{array}$ & 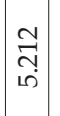 & 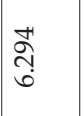 & $\begin{array}{l}\hat{0} \\
\infty \\
\sim \\
\stackrel{i}{\sim}\end{array}$ & 苻 & $\mid \begin{array}{c}0 \\
8 \\
10 \\
\\
\end{array}$ & 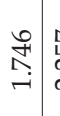 & $\begin{array}{l}\stackrel{\hat{D}}{N} \\
\stackrel{N}{N}\end{array}$ & 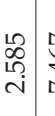 & 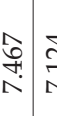 & 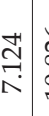 & 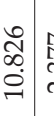 & $\begin{array}{l}\hat{N} \\
\text { ç } \\
\text { i }\end{array}$ & $\begin{array}{l}\stackrel{2}{\mathrm{~N}} \\
\mathrm{~N}\end{array}$ & 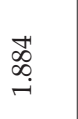 & 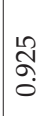 \\
\hline & 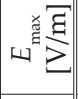 & 足 & 아 & $\begin{array}{l}\stackrel{2}{2} \\
\underset{\sigma}{+} \\
+\end{array}$ & 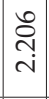 & $\stackrel{-}{\stackrel{-}{2}}$ & 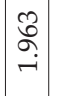 & 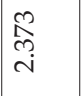 & 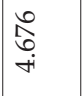 & $\begin{array}{l}\infty \\
10 \\
\infty \\
0 \\
0\end{array}$ & $\begin{array}{c}\hat{\omega} \\
\stackrel{m}{-} \\
-\end{array}$ & $\mid$\begin{tabular}{l}
1 \\
\hdashline \\
0 \\
0 \\
0
\end{tabular} & \begin{tabular}{c|c}
0 & \\
$\infty$ & \\
$\infty$ & \\
0 &
\end{tabular} & $\begin{array}{l}\text { 至 } \\
\text { Oे }\end{array}$ & \begin{tabular}{l|l}
0 & \multicolumn{2}{c}{} \\
$\infty$ & $g$ \\
$\dot{v}$ & \multicolumn{2}{c|}{}
\end{tabular} & $\begin{array}{l}\infty \\
\infty \\
\infty \\
+ \\
ن\end{array}$ & 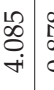 & 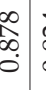 & $\begin{array}{c}\underset{N}{\mathbb{N}} \\
0 \\
0 \\
0\end{array}$ & $\begin{array}{l}\mathcal{N} \\
\infty \\
0 \\
0\end{array}$ & 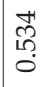 \\
\hline \multirow{3}{*}{ 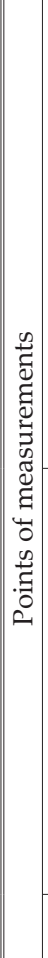 } & 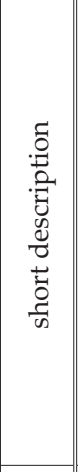 & 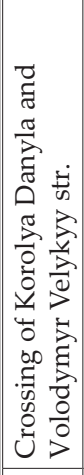 & 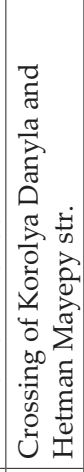 & 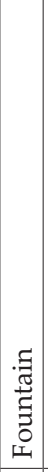 & 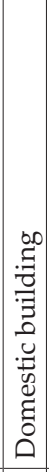 & 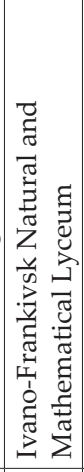 & 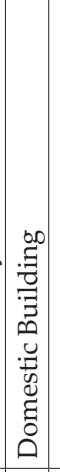 & 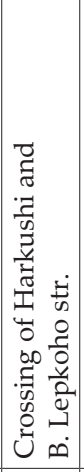 & 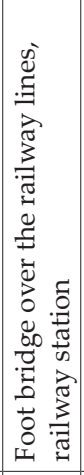 & 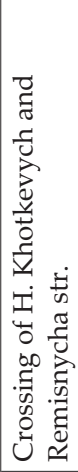 & 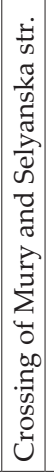 & 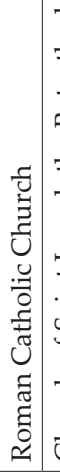 & 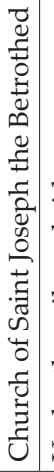 & 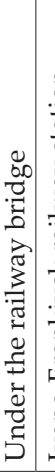 & 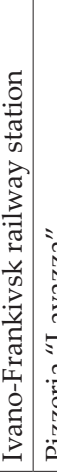 & 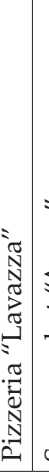 & 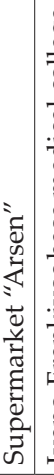 & 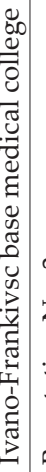 & 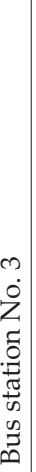 & 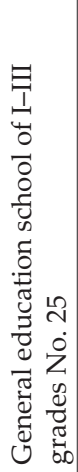 & 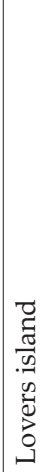 \\
\hline & 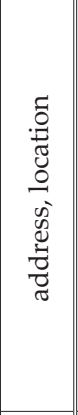 & 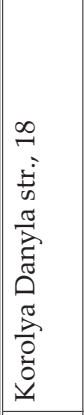 & 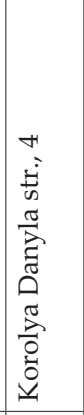 & 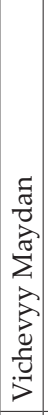 & 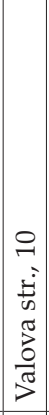 & 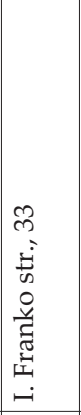 & 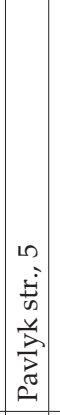 & 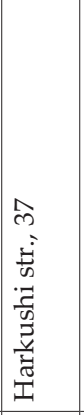 & 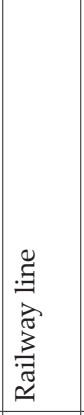 & 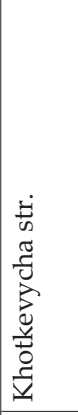 & 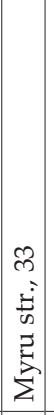 & 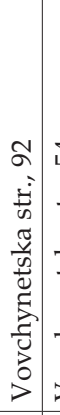 & 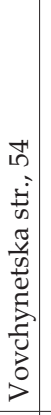 & 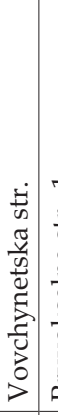 & 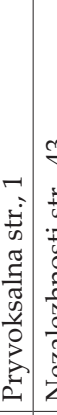 & 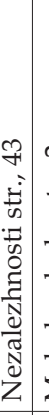 & 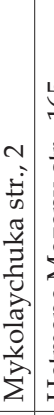 & 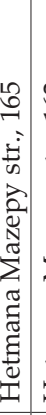 & 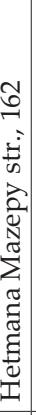 & 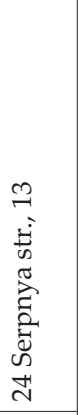 & 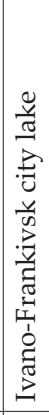 \\
\hline & $\dot{z}$ & $\hat{\mathrm{N}}$ & $\stackrel{\infty}{\wedge}$ & 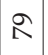 & $\infty$ & $\vec{\infty}$ & $\tilde{\infty}$ & $\infty$ & $\infty$ & 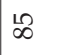 & $\infty$ & $\infty$ & $\infty$ & ळ & \& & $\bar{\sigma}$ & สু & $\alpha$ & ガ & ำ & ๙̊ \\
\hline
\end{tabular}




\begin{tabular}{|c|c|c|c|c|c|c|c|c|c|c|c|c|c|c|c|}
\hline 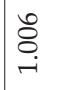 & $\begin{array}{l}\mathcal{O} \\
\delta \\
0\end{array}$ & ठิ & 足 & 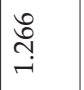 & $\begin{array}{c}m \\
\stackrel{f}{+} \\
0\end{array}$ & \begin{tabular}{l|}
$\infty$ \\
$\infty$ \\
0 \\
0 \\
0
\end{tabular} & $\begin{array}{l}0 \\
8 \\
0 \\
0\end{array}$ & & $\begin{array}{l}\hat{\sigma} \\
0 \\
0 \\
0\end{array}$ & $\begin{array}{l}\infty \\
\stackrel{\infty}{0} \\
\stackrel{0}{0} \\
0\end{array}$ & 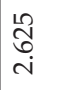 & 苟 & $\stackrel{\stackrel{2}{7}}{\stackrel{f}{0}}$ & Ő & 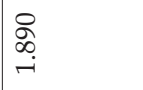 \\
\hline $\begin{array}{l}\hat{\sigma} \\
\text { ஸे } \\
\text { in }\end{array}$ & 号 & 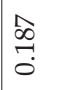 & $\begin{array}{l}\hat{\circ} \\
\stackrel{\circ}{\circ} \\
\wedge\end{array}$ & $\begin{array}{c}\infty \\
\infty \\
\infty \\
0 \\
0\end{array}$ & 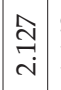 & $\begin{array}{c}\infty \\
\stackrel{\infty}{\sim} \\
\stackrel{-}{-}\end{array}$ & $\begin{array}{l}\widehat{\infty} \\
\infty \\
\infty \\
-\end{array}$ & $\underset{\text { 令 }}{\text { i }}$ & 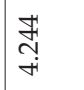 & 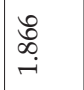 & $\begin{array}{l}\stackrel{1}{7} \\
\underset{\infty}{\infty}\end{array}$ & 怘 & $\begin{array}{l}\infty \\
\infty \\
\infty \\
+\end{array}$ & $\begin{array}{l}\text { 足 } \\
\stackrel{+}{1} \\
0\end{array}$ & 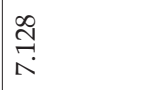 \\
\hline $\begin{array}{l}0 \\
0 \\
0 \\
0\end{array}$ & 苍 & 旾 & 足 & 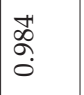 & $\begin{array}{c}\infty \\
\tilde{n} \\
\sim \\
i\end{array}$ & $\begin{array}{c}\hat{\alpha} \\
\infty \\
0 \\
0\end{array}$ & $\begin{array}{l}0 \\
\stackrel{0}{0} \\
0\end{array}$ & $\begin{array}{l}10 \\
\infty \\
\infty \\
0 \\
0\end{array}$ & 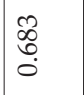 & $\begin{array}{l}\infty \\
\stackrel{\infty}{0} \\
\stackrel{0}{0}\end{array}$ & 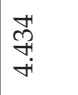 & $\begin{array}{c}\not 1 \\
\infty \\
0 \\
0\end{array}$ & 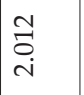 & 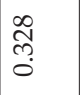 & הิ \\
\hline 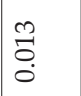 & 产 & $\begin{array}{l}\infty \\
0 \\
0 \\
0 \\
0\end{array}$ & $\begin{array}{l}\tilde{N} \\
\infty \\
\infty \\
\infty\end{array}$ & $\begin{array}{l}\vec{\sigma} \\
\ddot{\infty}\end{array}$ & $\begin{array}{l}\stackrel{\text { స్ }}{0} \\
0\end{array}$ & 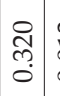 & $\begin{array}{l}0 \\
0 \\
0 \\
0\end{array}$ & Iี & $\stackrel{\text { I }}{\underset{7}{+}}$ & 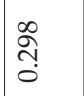 & $\begin{array}{l}19 \\
\infty \\
\infty \\
\infty \\
\infty\end{array}$ & 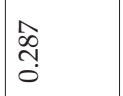 & & م. & \begin{tabular}{l}
3 \\
\multirow{3}{n}{} \\
10 \\
10
\end{tabular} \\
\hline 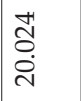 & $\begin{array}{l}10 \\
\infty 0 \\
0 \\
0\end{array}$ & 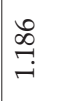 & $\begin{array}{l}0 \\
\widehat{U} \\
0 \\
0 \\
0\end{array}$ & $\begin{array}{l}\stackrel{8}{0} \\
\stackrel{1}{+} \\
\infty\end{array}$ & 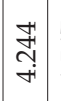 & 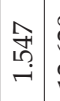 & 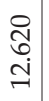 & 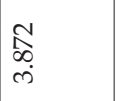 & $\begin{array}{l}\infty \\
\infty \\
0 \\
0\end{array}$ & $\underset{\sim}{\stackrel{\infty}{c}}$ & $\begin{array}{l}8 \\
0 \\
\infty \\
\infty \\
-1\end{array}$ & 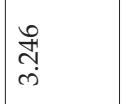 & 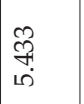 & 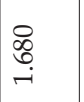 & $\begin{array}{l}\text { Iี } \\
\text { İ } \\
\text { I }\end{array}$ \\
\hline $\begin{array}{l}\infty \\
\infty \\
\infty \\
\wedge \\
\end{array}$ & $\begin{array}{l}n \\
\text { @ొ } \\
0 \\
0\end{array}$ & $\stackrel{\stackrel{2}{7}}{\stackrel{2}{0}}$ & $\begin{array}{l}\tilde{3} \\
\infty \\
\infty \\
\dot{c}\end{array}$ & 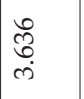 & $\begin{array}{l}m \\
\infty \\
0 \\
i\end{array}$ & $\begin{array}{c}0 \\
\infty \\
\infty \\
0\end{array}$ & $\begin{array}{l}\text { సิ } \\
\text { ڤ }\end{array}$ & $\stackrel{\vec{ন}}{\stackrel{\sim}{-}}$ & $\begin{array}{l}\text { ণิ } \\
\text { ஸे } \\
\text { ले }\end{array}$ & సิ & $\begin{array}{l}D \\
\stackrel{D}{0} \\
\text { N̦ }\end{array}$ & 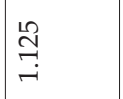 & 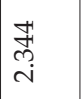 & 尽 & $\begin{array}{l}\text { 7ै } \\
\text { เก } \\
\text { மก }\end{array}$ \\
\hline 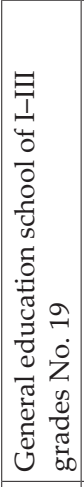 & 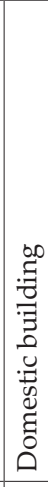 & 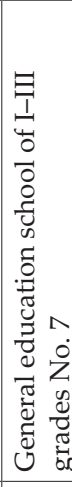 & 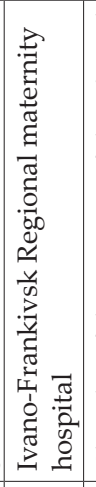 & 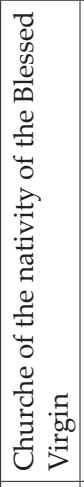 & 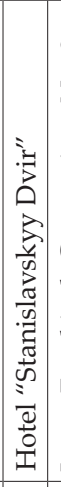 & 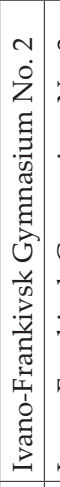 & 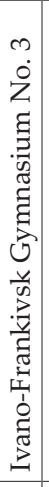 & 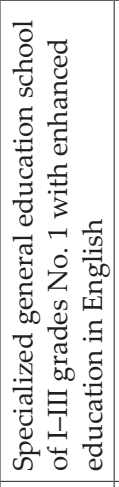 & 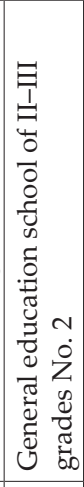 & 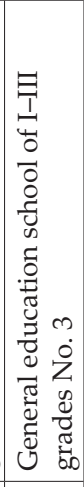 & 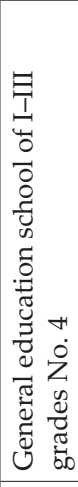 & 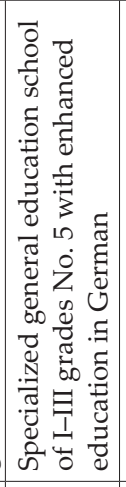 & 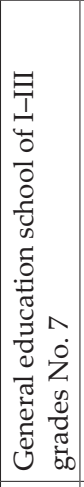 & 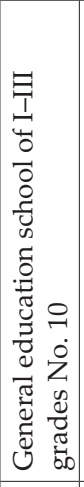 & 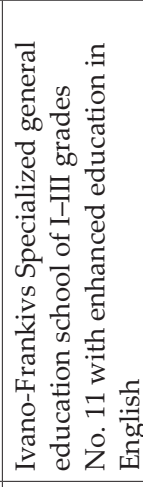 \\
\hline 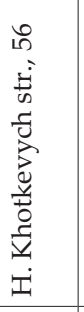 & 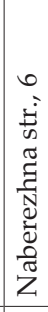 & 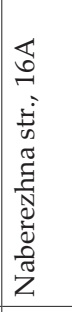 & 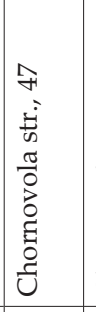 & 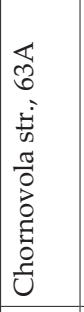 & 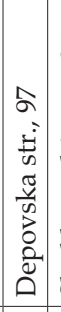 & 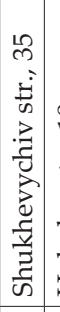 & 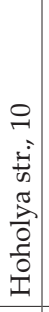 & 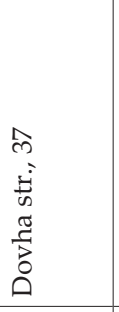 & 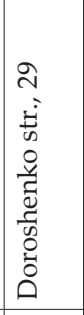 & 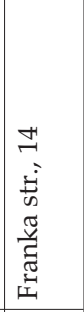 & 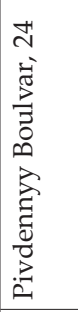 & 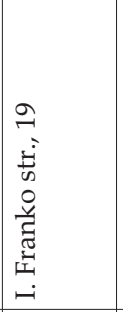 & 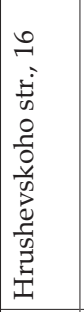 & 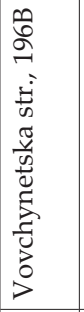 & 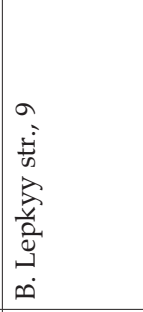 \\
\hline ลे & $\stackrel{\infty}{\circ}$ & ๙ & $\stackrel{8}{\circ}$ & $\underset{\sigma}{\sigma}$ & $\underset{\sigma}{\sigma}$ & 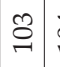 & $\underset{\sim}{\stackrel{C}{\prime}}$ & $\stackrel{2}{\stackrel{0}{-1}}$ & $\stackrel{\overbrace{}}{\circ}$ & $\stackrel{ }{\circ}$ & $\stackrel{\infty}{\stackrel{-}{2}}$ & $\stackrel{\text { Oे }}{1}$ & $\stackrel{ }{\Rightarrow}$ & $\exists$ & $\stackrel{\beth}{\exists}$ \\
\hline
\end{tabular}




\begin{tabular}{|c|c|c|c|c|c|c|c|c|c|c|c|}
\hline$\stackrel{\Xi}{\Xi}$ & 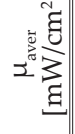 & $\begin{array}{l}\stackrel{0}{0} \\
\stackrel{0}{0}\end{array}$ & $\begin{array}{l}\hat{0} \\
\stackrel{0}{0}\end{array}$ & 苞 & $\begin{array}{l}\tilde{0} \\
\stackrel{0}{0}\end{array}$ & $\vec{~}$ & $\begin{array}{l}\infty \\
\stackrel{\infty}{0} \\
0\end{array}$ & $\begin{array}{l}\stackrel{0}{0} \\
\stackrel{0}{0}\end{array}$ & $\begin{array}{l}n \\
\stackrel{2}{0} \\
0 \\
0\end{array}$ & 胥 & $\begin{array}{l}\infty \\
\infty \\
\stackrel{\infty}{0}\end{array}$ \\
\hline 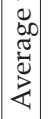 & $I^{(\underline{m}}$ & 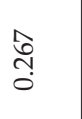 & 芯 & 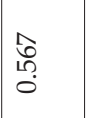 & 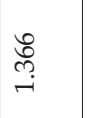 & $\underset{\vec{i}}{\stackrel{m}{i}}$ & 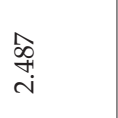 & 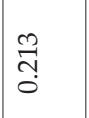 & 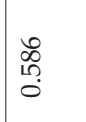 & 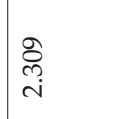 & $\begin{array}{l}\text { Pै } \\
\text { în } \\
\text { in }\end{array}$ \\
\hline & 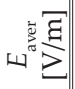 & $\begin{array}{l}\text { సิ } \\
\text { 웅 }\end{array}$ & $\begin{array}{l}\stackrel{0}{\infty} \\
\stackrel{\infty}{0} \\
0\end{array}$ & $\begin{array}{l}\infty \\
\infty \\
\stackrel{0}{0}\end{array}$ & $\stackrel{\stackrel{2}{7}}{\stackrel{2}{0}}$ & $\begin{array}{l}\vec{\infty} \\
\stackrel{0}{o}\end{array}$ & 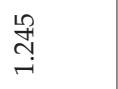 & त्ञ & 足 & $\begin{array}{l}\stackrel{0}{\infty} \\
\infty \\
\infty \\
0\end{array}$ & $\begin{array}{l}\widetilde{\sigma} \\
\sigma \\
\delta\end{array}$ \\
\hline 气 & 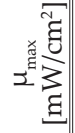 & ஜ: & 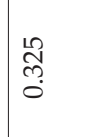 & 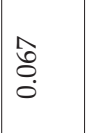 & \begin{tabular}{l}
$\stackrel{\infty}{*}$ \\
\hdashline
\end{tabular} & त्ञ & $\begin{array}{l}m \\
\stackrel{0}{0}\end{array}$ & $\begin{array}{l}0 \\
0 \\
0 \\
0 \\
0\end{array}$ & $\begin{array}{l}0 \\
\stackrel{0}{0} \\
0\end{array}$ & 命 & 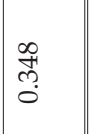 \\
\hline 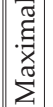 & 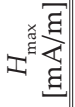 & 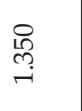 & $\stackrel{\stackrel{\mathscr{T}}{7}}{\underset{\sim}{\oplus}}$ & \begin{tabular}{l}
$\infty$ \\
\multirow{\infty}{\infty}{} \\
$\stackrel{-}{-}$
\end{tabular} & $\begin{array}{l}\stackrel{\hat{\infty}}{\infty} \\
\stackrel{\infty}{\rightarrow}\end{array}$ & $\begin{array}{l}\stackrel{\circ}{\circ} \\
\infty \\
i \\
i\end{array}$ & $\begin{array}{l}\tilde{\sigma} \\
+\end{array}$ & 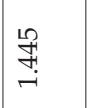 & 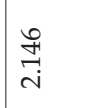 & 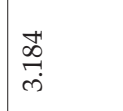 & 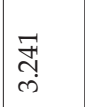 \\
\hline & 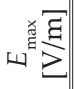 & 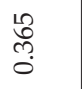 & 芯 & $\begin{array}{l}\text { Oे } \\
\text { مִ } \\
0\end{array}$ & $\begin{array}{l}\text { ô } \\
\stackrel{0}{0}\end{array}$ & 宊 & $\underset{\sim}{\vec{\Delta}}$ & $\begin{array}{l}2 \\
6 \\
0 \\
0\end{array}$ & 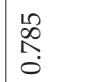 & ֻٕ & $\begin{array}{l}R \\
0 \\
\infty \\
\text { id }\end{array}$ \\
\hline & 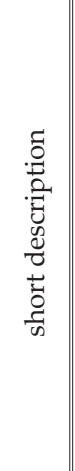 & 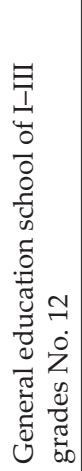 & 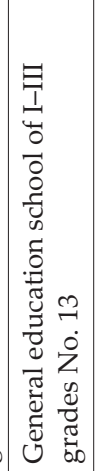 & 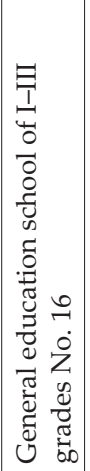 & 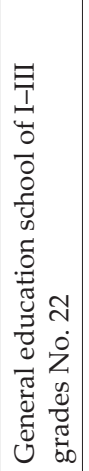 & 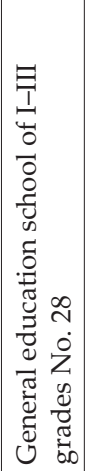 & 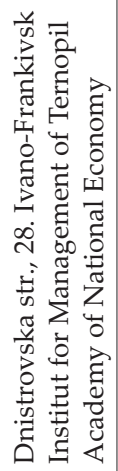 & 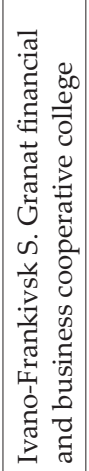 & 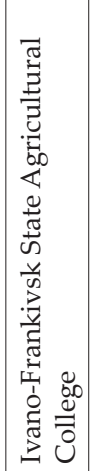 & 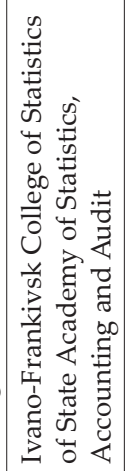 & 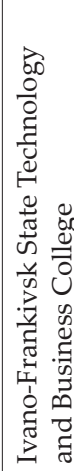 \\
\hline 2 & 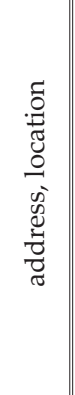 & 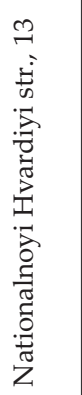 & 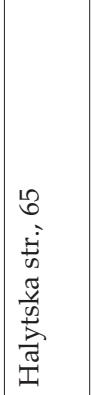 & 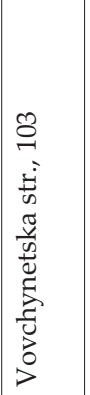 & 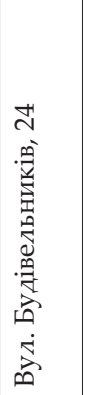 & 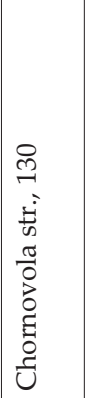 & 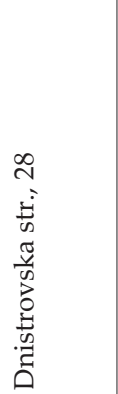 & 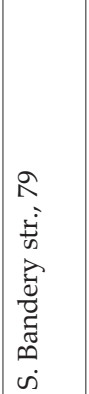 & 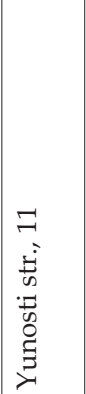 & 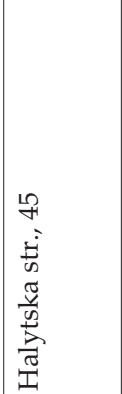 & 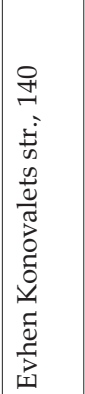 \\
\hline & $\dot{z}$ & $\stackrel{m}{\Rightarrow}$ & $\underset{\exists}{\exists}$ & $\stackrel{\mathscr{2}}{=}$ & $\stackrel{?}{=}$ & $\hat{\exists}$ & $\stackrel{\infty}{\exists}$ & $\stackrel{\partial}{\exists}$ & $\stackrel{\text { Iิ }}{\sim}$ & $\vec{I}$ & $\underset{\Xi}{ }$ \\
\hline
\end{tabular}




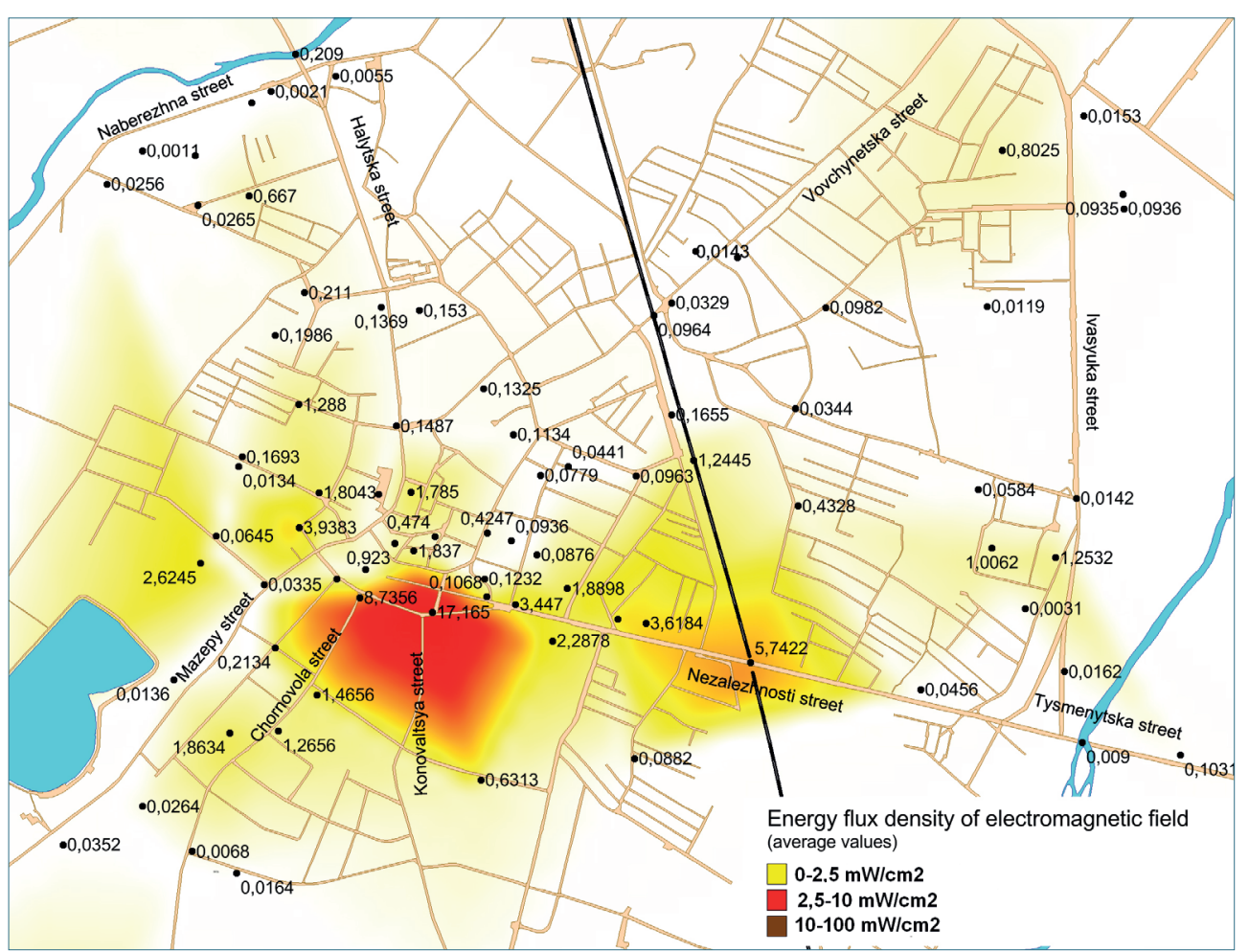

Fig. 1. Average values of surface energy flux density in Ivano-Frankivsk

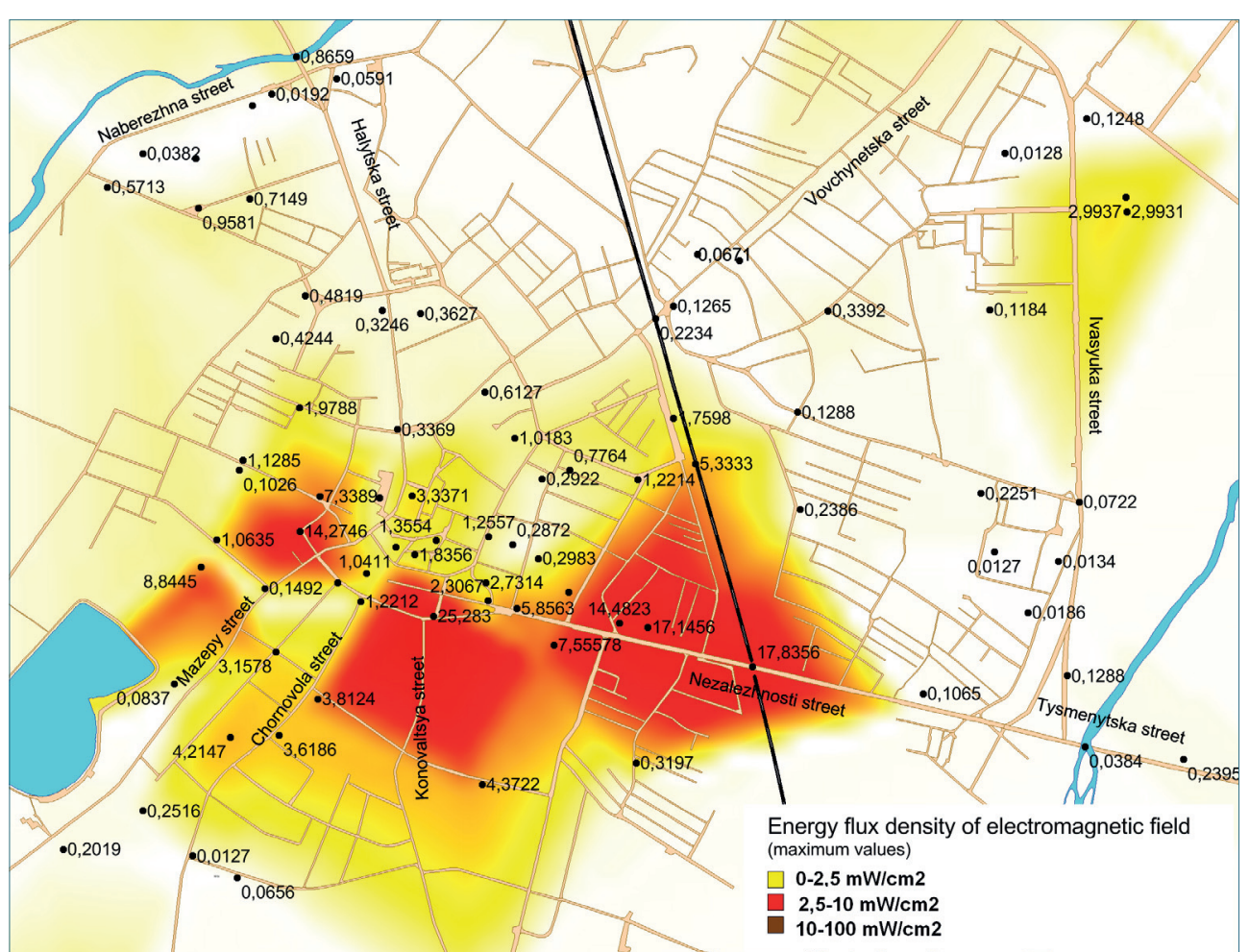

Fig. 2. Maximum values of surface energy flux density in Ivano-Frankivsk 


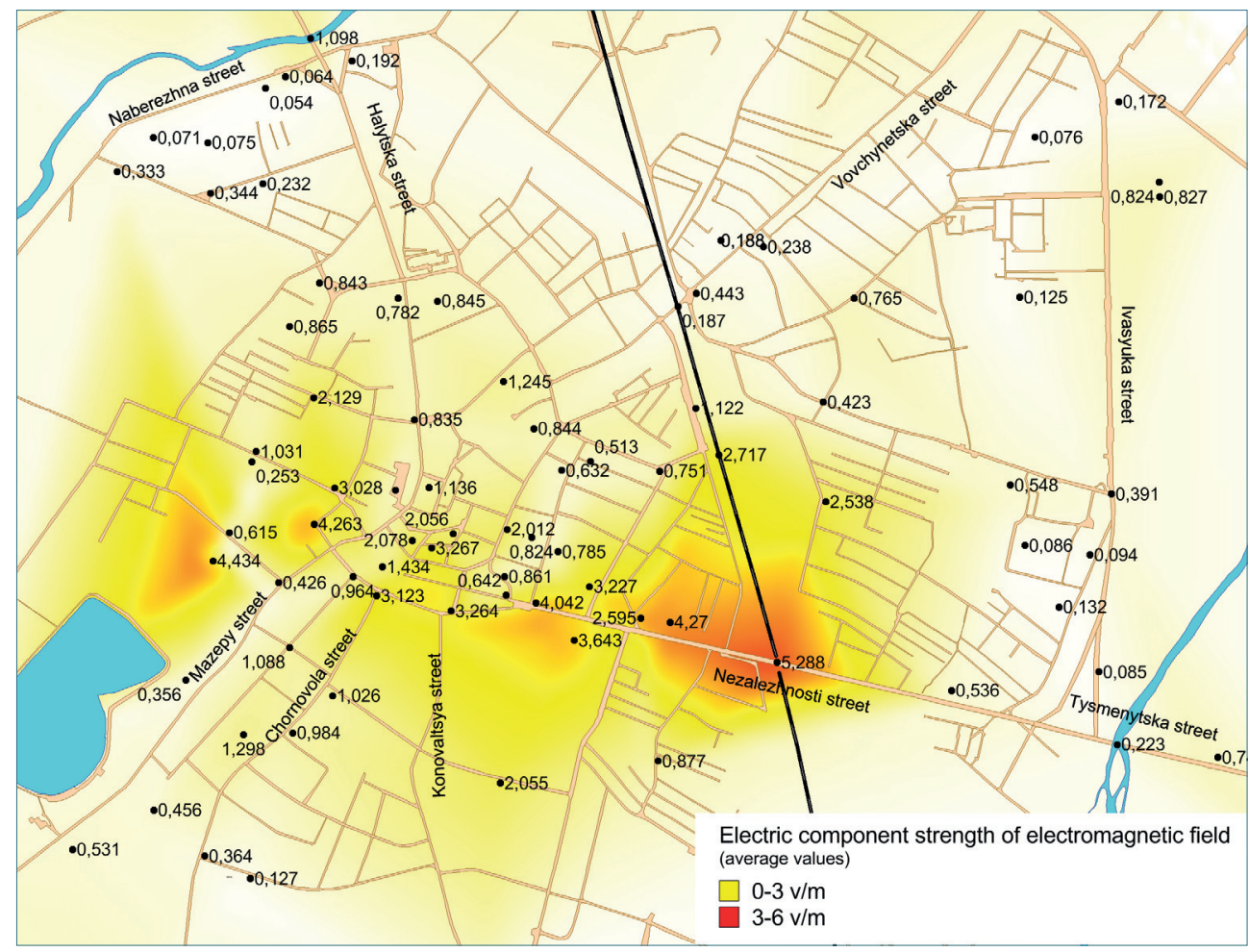

Fig. 3. Average values of electric component strength of electromagnetic field in Ivano-Frankivsk

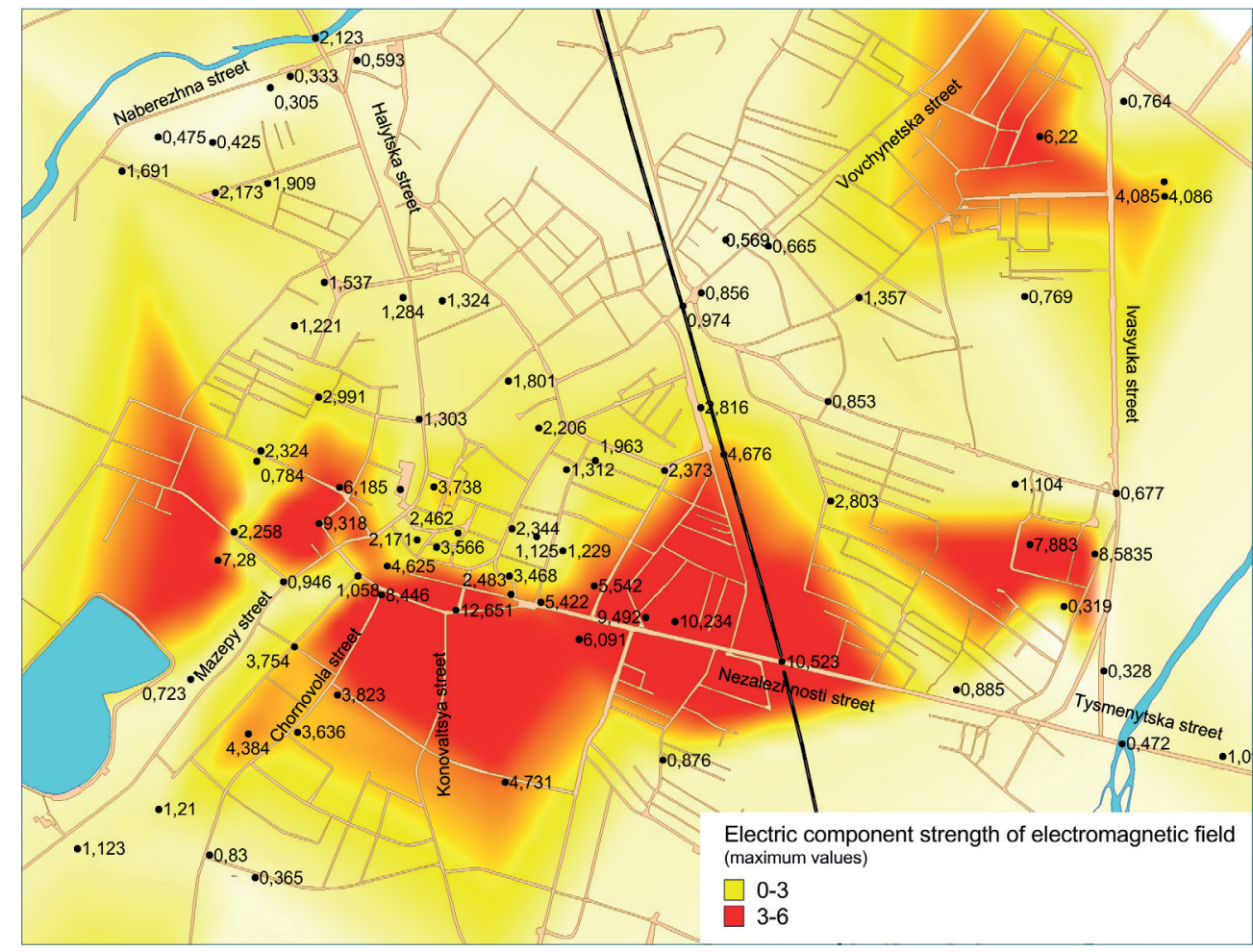

Fig. 4. Maximum values of the electric component strength of electromagnetic field in Ivano-Frankivsk 
So, in the urban social ecological system in Ivano-Frankivsk city is seen the elevation of maximum permissible limit for surface density of electromagnetic field power $\mu$ in terms of average values of this factor by 7 points of test area and is from $2.62 \mathrm{~mW} / \mathrm{cm}^{2}$ to $17.16 \mathrm{~mW} / \mathrm{cm}^{2}$, and in terms of the maximum values of this factor - by 22 points of test area, is from $2.73 \mathrm{~mW} / \mathrm{cm}^{2}$ to $25.28 \mathrm{~mW} / \mathrm{cm}^{2}$ (maximum permissible limit is $2.5 \mathrm{~mW} / \mathrm{cm}^{2}$ ). The average valu $\mu_{\text {aver }}$ in Ivano-Frankivsk city, is calculated taking into account the parameters $\mu_{\text {aver }}$ by all 122 points of test area and is $0.65 \mathrm{~mW} / \mathrm{cm}^{2}$, and the average value $\mu_{\text {max }}$ calculated taking into account the parameters $\mu_{\max }$ by all 122 points of test area, and is $1.73 \mathrm{~mW} / \mathrm{cm}^{2}$. The elevation of maximum permissible limit of electricical field intensity $\mathrm{E}$ in terms of average values of this factor is seen by 11 points of the test area, and is from $3.03 \mathrm{~V} / \mathrm{m}$ to $5.29 \mathrm{~V} / \mathrm{m}$, and in terms of the maximum values of this factor - by 29 points of the test area is from $3.08 \mathrm{~V} / \mathrm{m}$ to $12.65 \mathrm{~V} / \mathrm{m}$ (maximum permissible limit is $3 \mathrm{~V} / \mathrm{m}$ ). The average value of $E_{\text {aver }}$ in Ivano-Frankivsk city, calculated taking into account the parameters $E_{\text {aver }}$ by all 122 points of the test area, is $0.97 \mathrm{~V} / \mathrm{m}$ and the average value $E_{\max }$, calculated taking into account the parameters $E_{\max }$ by all 122 points of test area is $2.31 \mathrm{~V} / \mathrm{m}$.

\section{Methods of Medico-Ecological Researches}

Medical research shows that the most sensitive to the influence of EMF of industrial origin in the human body are the nervous, cardiovascular, endocrine, reproductive systems and the eyes, and EMF can also cause a significant influence on the metabolic rate $[1,2,4,6]$, encourage such syndrome as a medical phenomenon as a result of acute and chronic exposure of EMF of radio frequency range of nonlethal intensity equal to or above $10 \mathrm{~mW} / \mathrm{cm}^{2}$. To the symptoms belong reactions of the central nervous system and clinical signs of autonomic systems that are appear in changes in mood manipulations and behavior. Clinical signs are cardiovascular, gastrointestinal or endocrine nature [8]. Regulation of the heart functions in the human body is made through the local and central mechanisms. To the local regulation mechanisms apply myogenous, nervous and humoral, that are presented in the heart.

The myogenic regulatory mechanisms:

1) preloads - the more ventricle of heart is extended with running blood, the greater is his contraction power;

2) afterloads - increasing of contraction power of the left ventricle by increasing the diastolic pressure in the aorta;

3) rate of heart contraction - by increasing of the heart rate is growing up the power contraction.

The local nervous regulation mechanism of the heart functions is provided by the cardiac conduction system (sino-atrial node, intermodal atrial tracks, atrioventricular node, bundle of His branch block and its legs, Purkinje fibers), that is capable of impulsive rhythmic membrane depolarization, which leads to the generation of the action potential and reduction of cardiac muscle. 
The local humoral regulation of the heart functions consists in the heart synthesis of biologically active substances (natriuretic hormone, catecholamines, etc.), that are able to influence the contractile activity of the myocardium.

The central regulation mechanisms of the heart functions are provided by a nice branch of the nervous system (increases the power contraction of myocardium, increases heart rate, getting up speed of conduction of excitement by the leading heart system, increases the excitability of the heart) and parasympatic nervous system (reduces the power contraction of myocardium, reduces heart rate, reduces speed of conduction of excitement by the leading heart system, reduces the excitability of the heart). So, the regulation of the heart functions is determined by the cooperation of intracardiac and extracardiac mechanisms [10]. The humen cardiovascular system is characterized by adaptive meteotropic reactions. And the best adaptive reactions are typical for young people at the age of 18-25. By the medical investigations analysis of heart rate variability in young people it was found that the most resistant to various types of weather are young women, and the minor stress of adaptation mechanisms of the cardiovascular system appears by the II type of weather (atmosphere pressure change from 3.75 to $5.5 \mathrm{mmHg}$ a day, changing the average daily temperature at $5-10^{\circ} \mathrm{C}$, relative humidity at $60-85 \%$, low cyclonic activity) $[3,5]$.

Offered estimation method for environmental effects on the cardiovascular system, which is a sensitive indicator of violations of human physiological processes. The corresponding data were measured by the thermometer Omron M2 Basic. Into the base of the invention was taken the task to find a way which allows for identifying and investigating the impact of anthropogenic electromagnetic fields on the cardiovascular system of the people. The method is based on the observation of the human heart, which is within the territory of increased effect of EMF and for data comparison within the territory of the controled area where the impact of EMF does not exceed the norm. For conducting observations as a rate of arterial pressure $\varphi$ is convenient to use the share, which is reached by lower (diastolic) pressure $D$ in relation to the upper (systolic) $S: \varphi=D / S$ [7]. For the medical and environmental research at selected points of test area of urban area of Ivano-Frankivsk city (Tab. 1) at one time with intensity measurements of electric field $(E)$, magnetic field $(H)$ and the surface density of energy flow $(\mu)$ conducted measurements of heart rate $(H R)$, systolic arterial pressure $(S)$ and diastolic arterial pressure $(D)$. To determine the value of $S$ and $D$ and heart rate were involved young people at the age of 20-22, students of State higher education institution V. Stefanyk Carpathian National University. The indicators were determined separately for men and women at each point of the test area. The measurements were conducted five times at an intervals of 10 minutes separately by five men and five women. The determination conducted under meteorological conditions of type II. At each point of the test area was determined the arithmetic average value for $S$ and $D$ and heart rate separately for men and women. Based on the determined average values $S$ and $D$ at each point of the test area separately for men and women was determined the value of arterial pressure $\varphi$. The research results are presented in Table 2. 


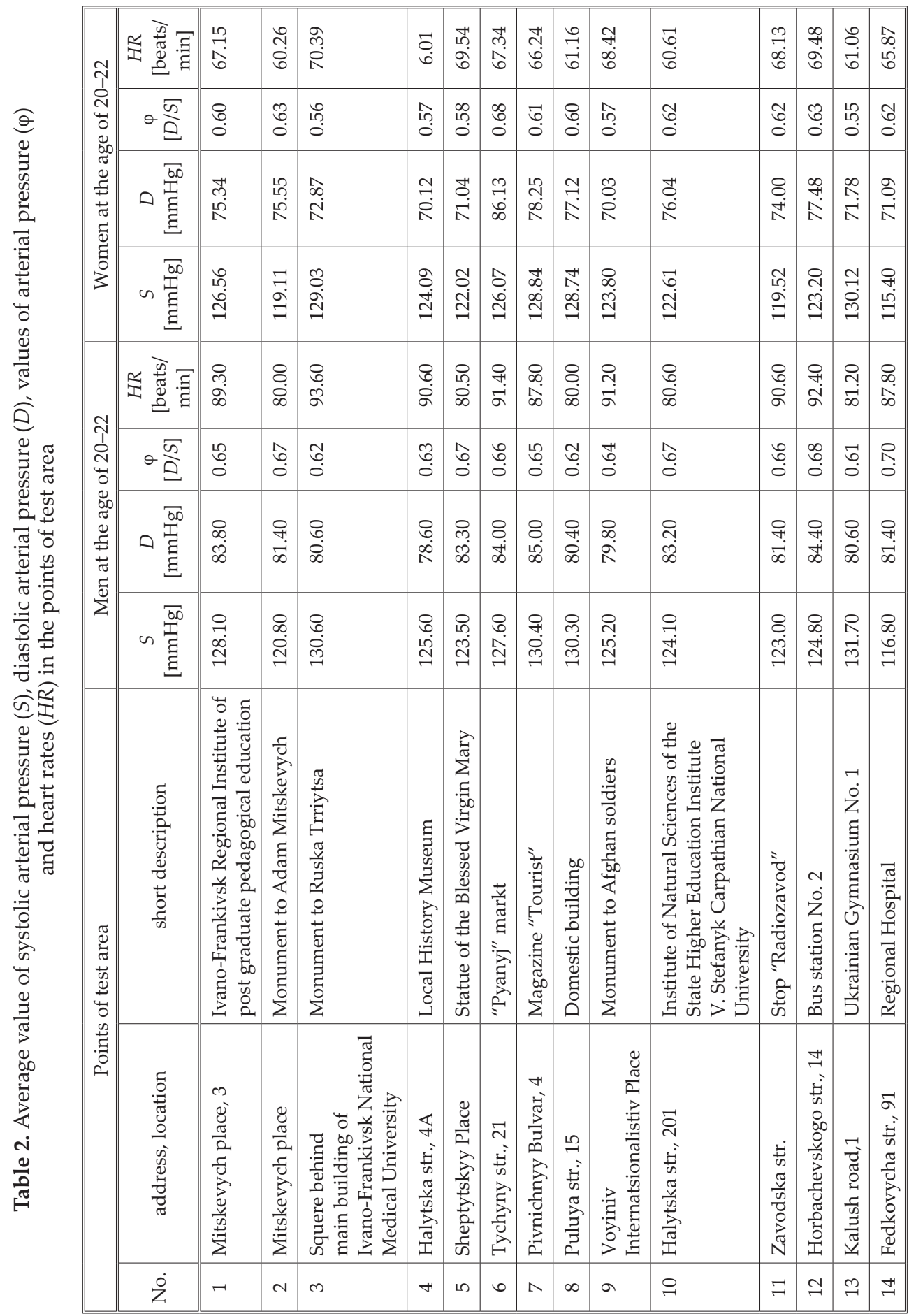




\begin{tabular}{|c|c|c|c|c|c|c|c|c|c|c|c|c|c|c|}
\hline \multirow{4}{*}{ 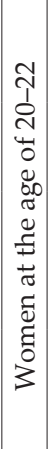 } & 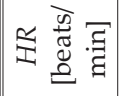 & 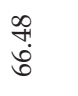 & $\begin{array}{l}\text { तु } \\
\qquad 80\end{array}$ & $\begin{array}{l}\infty \\
0 \\
10 \\
0\end{array}$ & $\begin{array}{l}\text { तิ } \\
\text { เู }\end{array}$ & $\begin{array}{l}\infty \\
\infty \\
10 \\
10\end{array}$ & नै & $\begin{array}{l}\text { הี } \\
\text { రే }\end{array}$ & $\begin{array}{l}8 \\
\infty \\
0\end{array}$ & $\begin{array}{l}\stackrel{2}{0} \\
\text { ஸे }\end{array}$ & $\begin{array}{l}0 \\
\infty \\
\infty \\
0\end{array}$ & $\begin{array}{l}0 \\
0 \\
\dot{0}\end{array}$ & $\begin{array}{l}\overrightarrow{5} \\
\stackrel{8}{0}\end{array}$ & 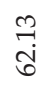 \\
\hline & $\theta \frac{\bar{\sigma}}{\theta}$ & ถึ. & $\begin{array}{l}\hat{\theta} \\
\dot{0}\end{array}$ & 艿 & $\stackrel{\circ}{\circ}$ & $\stackrel{\vec{b}}{0}$ & 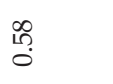 & ถึ. & ถึ. & 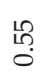 & రై & 占 & 艿 & مै \\
\hline & 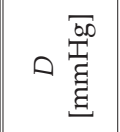 & $\begin{array}{l}\hat{\infty} \\
\stackrel{0}{0} \\
\stackrel{0}{0}\end{array}$ & 官 & مि & 굼 & $\stackrel{\circ}{\circ}$ & $\stackrel{\overbrace{}}{\overparen{R}}$ & సै: & 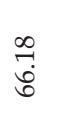 & $\stackrel{\infty}{\stackrel{\infty}{b}}$ & $\stackrel{+}{0}$ & $\begin{array}{l}\infty \\
\infty \\
\text { } \\
0\end{array}$ & $\stackrel{\Xi}{\circ}$ & $\begin{array}{l}\infty \\
\infty \\
0\end{array}$ \\
\hline & 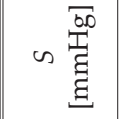 & 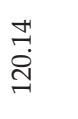 & $\begin{array}{l}\infty \\
1 \\
\stackrel{1}{=} \\
=\end{array}$ & $\begin{array}{l}\stackrel{\sim}{Z} \\
\stackrel{=}{=}\end{array}$ & $\begin{array}{l}\stackrel{\sigma}{O} \\
\stackrel{+}{ \pm} \\
=\end{array}$ & $\begin{array}{l}8 \\
10 \\
-1\end{array}$ & $\stackrel{M}{\stackrel{M}{二}}$ & 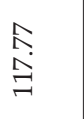 & $\begin{array}{l}\stackrel{\hat{n}}{\mathrm{i}} \\
\stackrel{\exists}{\exists}\end{array}$ & $\begin{array}{l}\stackrel{7}{7} \\
\stackrel{9}{=} \\
=\end{array}$ & $\begin{array}{l}\underset{J}{ } \\
\stackrel{\text { I }}{\exists}\end{array}$ & 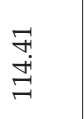 & $\begin{array}{l}\stackrel{\Xi}{6} \\
\stackrel{\Xi}{\Xi}\end{array}$ & $\begin{array}{l}\infty \\
\infty \\
\stackrel{0}{0}\end{array}$ \\
\hline \multirow{4}{*}{ 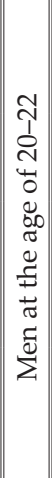 } & 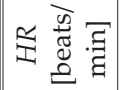 & $\begin{array}{l}\infty \\
\infty \\
\infty\end{array}$ & 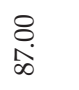 & 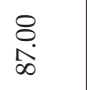 & $\begin{array}{l}\infty \\
\infty \\
\infty\end{array}$ & $\begin{array}{l}8 \\
0 \\
\infty \\
\infty\end{array}$ & $\begin{array}{l}8 \\
\text { مे }\end{array}$ & $\begin{array}{l}\text { 我 } \\
\text { வ }\end{array}$ & 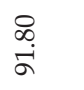 & $\begin{array}{l}\stackrel{8}{6} \\
\dot{+}\end{array}$ & $\begin{array}{l}\infty \\
\infty \\
\infty\end{array}$ & $\begin{array}{l}8 \\
\infty \\
\infty \\
\infty\end{array}$ & $\begin{array}{l}\text { † } \\
\text { হ }\end{array}$ & 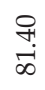 \\
\hline & $\odot \frac{\bar{\sigma}}{\theta}$ & $\stackrel{\bullet}{\circ}$ & $\begin{array}{l}\text { R } \\
0\end{array}$ & $\hat{0}$ & $\stackrel{M}{\hat{0}}$ & $\hat{0}$ & 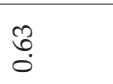 & $\underset{0}{\sigma}$ & $\underset{0}{\sigma}$ & $\begin{array}{l}\text { Jర } \\
0\end{array}$ & $\underset{O}{0}$ & $\stackrel{\vec{\sigma}}{0}$ & $\begin{array}{l}18 \\
0 \\
0\end{array}$ & $\begin{array}{l}0 \\
0 \\
0\end{array}$ \\
\hline & ○ & $\begin{array}{l}\text { సి } \\
\stackrel{\infty}{ }\end{array}$ & $\begin{array}{l}\text { P } \\
\text { i }\end{array}$ & $\begin{array}{l}\text { तิ } \\
\text { ळे }\end{array}$ & $\begin{array}{l}8 \\
\stackrel{\infty}{\infty}\end{array}$ & $\begin{array}{l}8 \\
\stackrel{0}{1}\end{array}$ & $\begin{array}{l}\stackrel{8}{\infty} \\
\stackrel{1}{N}\end{array}$ & तి & 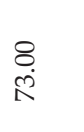 & $\begin{array}{l}8 \\
\stackrel{1}{x}\end{array}$ & $\begin{array}{l}\text { ํ } \\
\text { i }\end{array}$ & 异 & 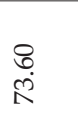 & 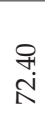 \\
\hline & $\omega \frac{\overline{b 0}}{\bar{E}}$ & $\begin{array}{l}\stackrel{8}{0} \\
\stackrel{\text { I }}{7}\end{array}$ & $\begin{array}{l}\text { त् } \\
\infty \\
\stackrel{\infty}{=}\end{array}$ & $\begin{array}{l}8 \\
0 \\
= \\
=\end{array}$ & 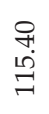 & $\begin{array}{l}9 \\
9 \\
\stackrel{1}{1} \\
=\end{array}$ & 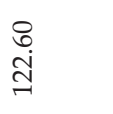 & $\begin{array}{l}\text { ָุ } \\
\stackrel{-}{=}\end{array}$ & $\begin{array}{l}8 \\
\stackrel{0}{7}\end{array}$ & $\begin{array}{l}\infty \\
\infty \\
\stackrel{+}{+} \\
\exists\end{array}$ & $\begin{array}{l}\stackrel{9}{+} \\
\stackrel{0}{=}\end{array}$ & $\begin{array}{l}\infty \\
\infty \\
\stackrel{1}{=} \\
=\end{array}$ & $\begin{array}{l}8 \\
\stackrel{8}{=} \\
\stackrel{1}{=}\end{array}$ & त్ \\
\hline \multirow{3}{*}{ 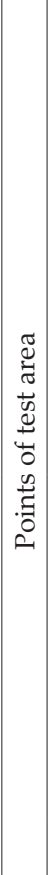 } & 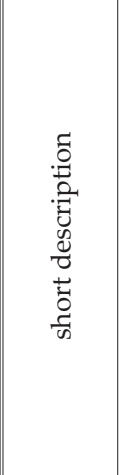 & 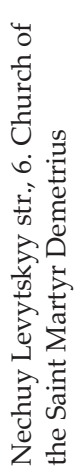 & 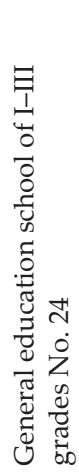 & 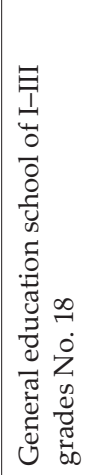 & 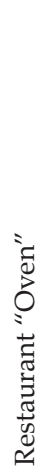 & 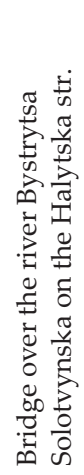 & 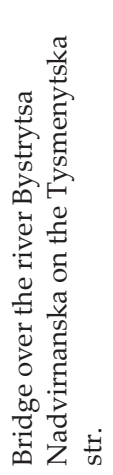 & 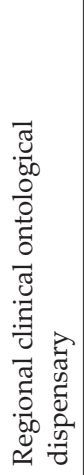 & 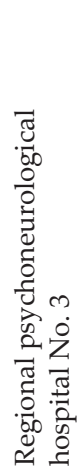 & 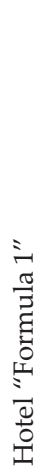 & 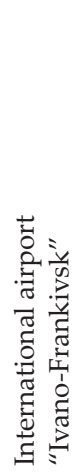 & 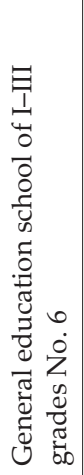 & 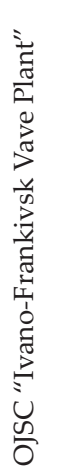 & 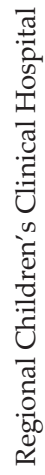 \\
\hline & 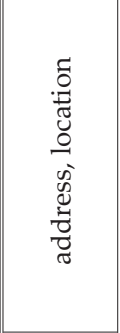 & 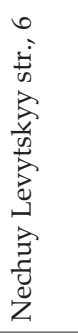 & 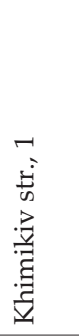 & 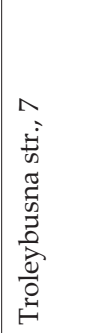 & 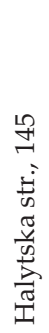 & 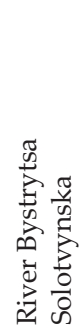 & 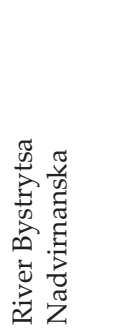 & 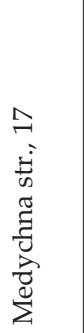 & 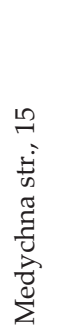 & 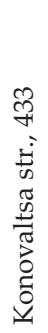 & 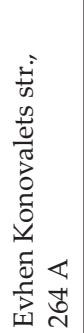 & 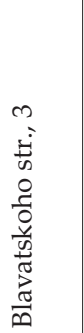 & 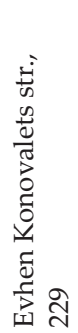 & 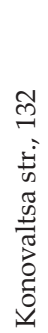 \\
\hline & Z & $\stackrel{10}{\sim}$ & $\stackrel{\bullet}{\sim}$ & $\approx$ & $\stackrel{\infty}{\sim}$ & $\stackrel{\curvearrowright}{2}$ & $\widetilde{N}$ & $\vec{\lambda}$ & $\approx$ & $\ddot{\sim}$ & $\stackrel{\Delta}{N}$ & $\stackrel{\llcorner 0}{\sim}$ & $\stackrel{\sim}{N}$ & $\widehat{\curvearrowright}$ \\
\hline
\end{tabular}




\begin{tabular}{|c|c|c|c|c|c|c|c|c|c|c|c|c|c|c|c|c|c|}
\hline $\begin{array}{l}\text { ले } \\
\text { सुं }\end{array}$ & $\begin{array}{l}8 \\
\stackrel{1}{1} \\
\stackrel{1}{*}\end{array}$ & $\begin{array}{l}\infty \\
\infty \\
\aleph \\
\aleph\end{array}$ & $\begin{array}{l}\text { ț } \\
\text { هి }\end{array}$ & $\begin{array}{l}\text { กิ } \\
\text { తెర }\end{array}$ & $\begin{array}{l}\overrightarrow{\hat{~}} \\
\text { దิ }\end{array}$ & $\begin{array}{l}\text { సે. } \\
\text { ถิ }\end{array}$ & 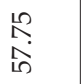 & $\begin{array}{l}\text { 웅 } \\
\text { 요 }\end{array}$ & $\begin{array}{l}\text { ले } \\
\ddot{n}\end{array}$ & $\begin{array}{l}\stackrel{\circ}{\infty} \\
\stackrel{1}{10}\end{array}$ & $\begin{array}{l}\qquad 0 \\
0 \\
\infty \\
0\end{array}$ & $\begin{array}{l}0 \\
\infty \\
\infty \\
\infty\end{array}$ & $\begin{array}{l}\text { तి } \\
\text { ம் }\end{array}$ & $\begin{array}{l}\stackrel{3}{0} \\
\stackrel{\leftrightarrow}{0}\end{array}$ & 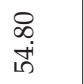 & $\begin{array}{l}\bar{N} \\
\text { న }\end{array}$ & 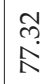 \\
\hline $\begin{array}{l}\infty \\
\stackrel{\infty}{\llcorner} \\
0\end{array}$ & ஸึ่ & ర్రా & $\stackrel{\text { பூ }}{\circ}$ & ت্ర & مि & $\begin{array}{l}\tilde{b} \\
\stackrel{0}{0}\end{array}$ & \begin{tabular}{c}
$\infty$ \\
$\stackrel{\infty}{\leftrightarrow 0}$ \\
\hdashline
\end{tabular} & Lొ & กิ & 今े & $\begin{array}{l}\tilde{U} \\
0 \\
0\end{array}$ & $\begin{array}{c}\infty \\
\stackrel{\infty}{10} \\
0\end{array}$ & $\begin{array}{l}\vec{b} \\
\stackrel{0}{0}\end{array}$ & $\begin{array}{l}\text { L̊? } \\
\stackrel{0}{0}\end{array}$ & $\stackrel{\mathscr{P}}{\stackrel{0}{0}}$ & 華 & గึ? \\
\hline 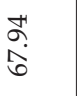 & $\begin{array}{l}\stackrel{2}{2} \\
\stackrel{6}{ }\end{array}$ & $\begin{array}{l}\infty \\
\infty \\
\infty \\
\stackrel{R}{R}\end{array}$ & $\begin{array}{l}\hat{N} \\
120 \\
\infty\end{array}$ & $\stackrel{0}{\stackrel{1}{\wedge}}$ & $\begin{array}{l}\stackrel{0}{\sim} \\
\stackrel{N}{N}\end{array}$ & 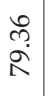 & 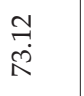 & 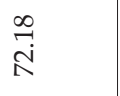 & $\begin{array}{l}\stackrel{M}{ت} \\
\stackrel{B}{0}\end{array}$ & $\begin{array}{l}\alpha \\
\alpha \\
\infty \\
i n\end{array}$ & $\begin{array}{c}\mathfrak{T} \\
\stackrel{2}{2} \\
\stackrel{2}{2}\end{array}$ & 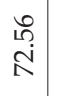 & $\begin{array}{l}\stackrel{+}{0} \\
\infty \\
\infty\end{array}$ & $\begin{array}{l}\text { के. } \\
\text { వి }\end{array}$ & $\begin{array}{l}\infty \\
\infty \\
\stackrel{+}{1}\end{array}$ & $\stackrel{10}{\stackrel{10}{+}}$ & $\begin{array}{l}\infty \\
\infty \\
0\end{array}$ \\
\hline $\begin{array}{l}\hat{\sigma} \\
\hat{ٍ} \\
ٍ\end{array}$ & $\begin{array}{l}\stackrel{f}{f} \\
\stackrel{一}{F}\end{array}$ & $\begin{array}{l}\stackrel{8}{0} \\
\stackrel{1}{\mid} \\
=\end{array}$ & $\begin{array}{l}\text { ఫे } \\
\text { నิ } \\
\stackrel{2}{\longrightarrow}\end{array}$ & 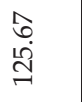 & $\begin{array}{l}\qquad 0 \\
\stackrel{0}{0} \\
\hat{\Xi}\end{array}$ & 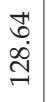 & $\begin{array}{l}\text { L } \\
\infty \\
\stackrel{0}{0} \\
\text { I }\end{array}$ & 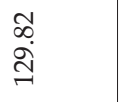 & $\begin{array}{l}\infty \\
\infty \\
\stackrel{J}{\beth} \\
=\end{array}$ & 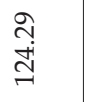 & 足 & 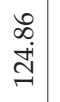 & 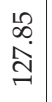 & $\begin{array}{l}\infty \\
\stackrel{1}{+} \\
\stackrel{+}{\simeq}\end{array}$ & 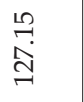 & $\begin{array}{l}8 \\
\stackrel{8}{10} \\
=\end{array}$ & $\stackrel{\triangle}{\vec{\Xi}}$ \\
\hline $\begin{array}{l}\text { 유 } \\
\text { ti }\end{array}$ & $\begin{array}{l}\text { ते } \\
\text { જે }\end{array}$ & $\begin{array}{l}8 \\
\text { ஸ́ }\end{array}$ & $\begin{array}{l}\text { ๖ } \\
\text { ๙ે }\end{array}$ & $\begin{array}{l}\stackrel{0}{+} \\
\text { + }\end{array}$ & \begin{tabular}{l}
$\circ$ \\
\multirow{2}{2}{} \\
$\circ$
\end{tabular} & 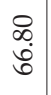 & 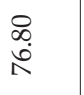 & $\begin{array}{l}8 \\
\stackrel{2}{1} \\
\text { In }\end{array}$ & $\begin{array}{l}\text { तิ } \\
\text { gิ }\end{array}$ & $\begin{array}{l}8 \\
8 \\
0\end{array}$ & §a & $\begin{array}{l}8 \\
\stackrel{1}{1} \\
\hat{N}\end{array}$ & $\begin{array}{l}\stackrel{8}{0} \\
\stackrel{+}{N}\end{array}$ & 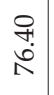 & $\begin{array}{l}\stackrel{8}{0} \\
\stackrel{i}{i}\end{array}$ & $\begin{array}{l}\text { 뭉 } \\
\stackrel{0}{0}\end{array}$ & 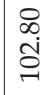 \\
\hline $\begin{array}{l}\text { ஜூ } \\
0\end{array}$ & $\begin{array}{l}+ \\
\stackrel{+}{0} \\
\stackrel{0}{*}\end{array}$ & $\begin{array}{l}\stackrel{0}{0} \\
\stackrel{0}{0}\end{array}$ & ণु & $\stackrel{\hat{\sigma}}{0}$ & $\begin{array}{l}\overrightarrow{6} \\
\dot{0}\end{array}$ & $\begin{array}{l}\mathscr{B} \\
\ddot{0}\end{array}$ & ஜூ & $\underset{\bullet}{\sigma}$ & $\underset{\bullet}{\overrightarrow{0}}$ & $\stackrel{\overrightarrow{6}}{\circ}$ & $\mid \begin{array}{l}\infty \\
0 \\
0 \\
0\end{array}$ & $\begin{array}{l}\text { ठே } \\
\stackrel{0}{0}\end{array}$ & 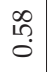 & $\begin{array}{l}8 \\
\stackrel{0}{0}\end{array}$ & $\stackrel{8}{\circ}$ & ठே & : \\
\hline $\begin{array}{l}\stackrel{8}{0} \\
\stackrel{1}{1}\end{array}$ & $\begin{array}{l}8 \\
\stackrel{0}{\circ} \\
\end{array}$ & $\begin{array}{l}\stackrel{8}{0} \\
\stackrel{1}{1}\end{array}$ & 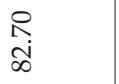 & $\begin{array}{l}\text { तิ } \\
\stackrel{\infty}{\infty}\end{array}$ & $\begin{array}{l}9 \\
9 \\
2\end{array}$ & 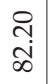 & 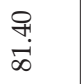 & $\begin{array}{l}\infty \\
\infty \\
\stackrel{x}{1}\end{array}$ & $\begin{array}{l}8 \\
\stackrel{0}{1} \\
\stackrel{1}{*}\end{array}$ & $\frac{8}{1}$ & $\begin{array}{c}8 \\
\dot{1} \\
\infty\end{array}$ & $\begin{array}{l}\infty \\
\dot{\infty} \\
\dot{\infty}\end{array}$ & 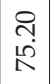 & 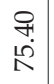 & $\begin{array}{l}8 \\
\stackrel{1}{1}\end{array}$ & 吕 & 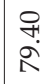 \\
\hline $\begin{array}{l}\text { 유 } \\
\text { مे } \\
\Rightarrow\end{array}$ & $\begin{array}{l}8 \\
\stackrel{0}{7} \\
=\end{array}$ & $\begin{array}{l}\stackrel{8}{\circ} \\
\stackrel{1}{\sigma}\end{array}$ & 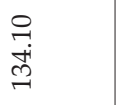 & $\begin{array}{l}\text { 운 } \\
\text { Â }\end{array}$ & $\begin{array}{l}\stackrel{1}{1} \\
\grave{1}\end{array}$ & 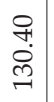 & $\begin{array}{l}\text { 울 } \\
\text { ô } \\
\text { I }\end{array}$ & $\begin{array}{l}\stackrel{9}{+} \\
\vec{m}\end{array}$ & 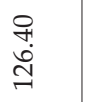 & $\begin{array}{l}\infty \\
\stackrel{1}{ } \\
\stackrel{1}{\mathbb{N}}\end{array}$ & $\begin{array}{l}8 \\
\stackrel{2}{0} \\
\stackrel{1}{1}\end{array}$ & $\begin{array}{c}\stackrel{+}{1} \\
\infty \\
\stackrel{0}{*}\end{array}$ & 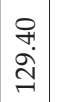 & 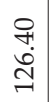 & $\begin{array}{l}\text { 울 } \\
\text { তิ }\end{array}$ & $\begin{array}{l}\stackrel{0}{7} \\
\stackrel{6}{=} \\
=\end{array}$ & 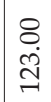 \\
\hline 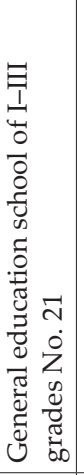 & 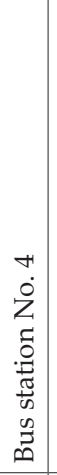 & 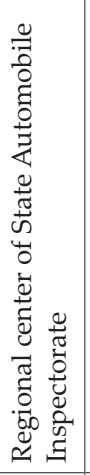 & 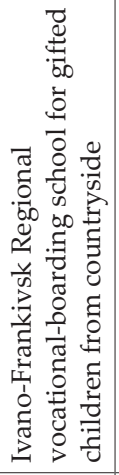 & 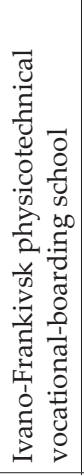 & 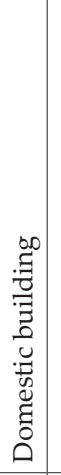 & 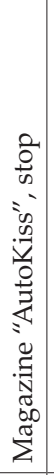 & 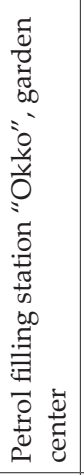 & 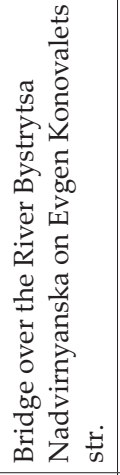 & 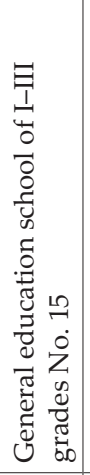 & 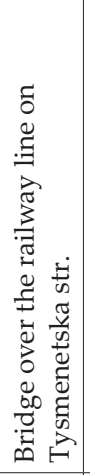 & 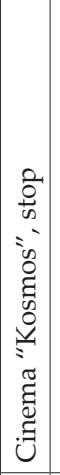 & 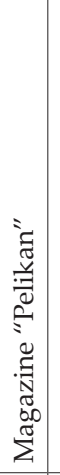 & 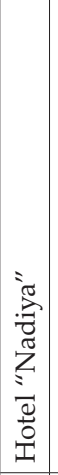 & 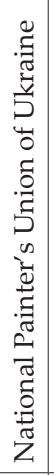 & 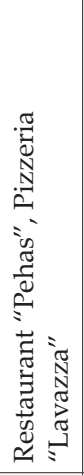 & 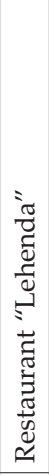 & 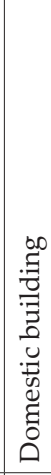 \\
\hline 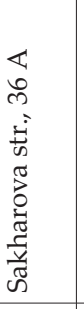 & 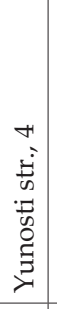 & 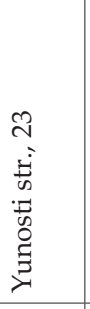 & 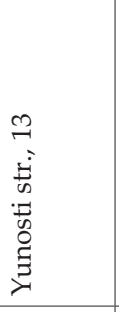 & 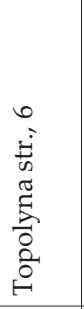 & 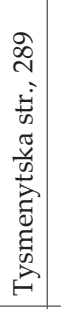 & 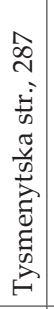 & 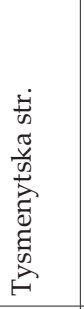 & 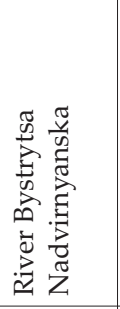 & 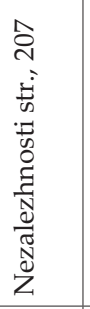 & 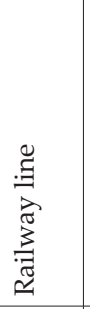 & 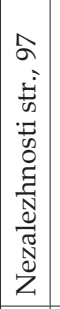 & 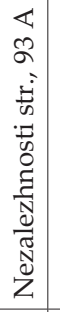 & 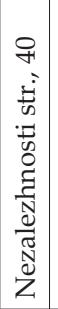 & 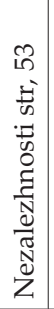 & 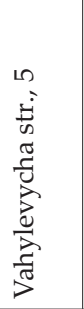 & 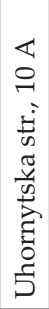 & 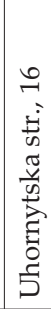 \\
\hline$\stackrel{\sim}{\sim}$ & ৯े & లి & $\bar{m}$ & $m$ & $m$ & ले & $\stackrel{10}{\infty}$ & లా & ले & $\infty$ & ले & 우 & $F$ & 구 & $\mathscr{P}$ & 标 & I8 \\
\hline
\end{tabular}




\begin{tabular}{|c|c|c|c|c|c|c|c|c|c|c|c|c|c|c|c|c|c|}
\hline \multirow{4}{*}{ 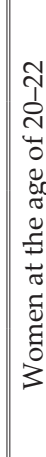 } & 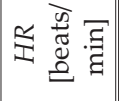 & $\begin{array}{l}\text { R } \\
\text { n }\end{array}$ & సุ. & $\begin{array}{l}\infty \\
\stackrel{\infty}{N} \\
\mathbb{N}\end{array}$ & $\begin{array}{l}\tilde{N} \\
\mathbb{N} \\
\end{array}$ & $\begin{array}{l}\mathbb{N} \\
\infty \\
0 \\
0\end{array}$ & 菅 & तु & 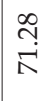 & $\begin{array}{l}\infty \\
10 \\
\infty \\
0 \\
0\end{array}$ & กิ & $\begin{array}{l}\mathfrak{c} \\
\infty \\
\infty \\
10\end{array}$ & $\begin{array}{l}8 \\
\text { in } \\
\text { in }\end{array}$ & $\begin{array}{l}\text { S } \\
10 \\
N\end{array}$ & 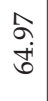 & $\begin{array}{l}\infty \\
i \\
\infty \\
0\end{array}$ & $\begin{array}{l}\tilde{\sigma} \\
\tilde{\sigma} \\
+\end{array}$ \\
\hline & $\theta \frac{\bar{\sigma}}{\varrho}$ & م̂̊ & 尚 & L̊ & $\begin{array}{l}\infty \\
\stackrel{\infty}{0} \\
\stackrel{0}{0}\end{array}$ & $\begin{array}{l}\mathbf{J} \\
0 \\
0\end{array}$ & $\stackrel{8}{0}$ & $\stackrel{8}{0}$ & ถู & $\stackrel{8}{0}$ & $\stackrel{8}{0}$ & $\begin{array}{l}\tilde{b} \\
0\end{array}$ & เִ & $\vec{b}$ & $\begin{array}{l}3 \\
0 \\
0\end{array}$ & ర్ & $\begin{array}{l}\infty \\
\stackrel{\infty}{0} \\
0\end{array}$ \\
\hline & 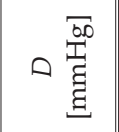 & $\begin{array}{l}\sigma \\
\dot{0}\end{array}$ & $\begin{array}{l}7 \\
\stackrel{n}{\infty}\end{array}$ & 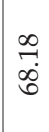 & $\stackrel{\text { I }}{\overrightarrow{1}}$ & $\begin{array}{l}0 \\
\stackrel{1}{1} \\
\end{array}$ & $\begin{array}{l}8 \\
\stackrel{0}{0} \\
0\end{array}$ & $\begin{array}{l}\vec{N} \\
\infty \\
\infty\end{array}$ & 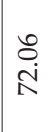 & $\stackrel{\infty}{\stackrel{\infty}{\infty}}$ & $\stackrel{ }{\stackrel{1}{1}}$ & 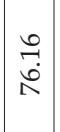 & $\begin{array}{c}\infty \\
\infty \\
\mathbb{N}\end{array} \mid$ & 永 & $\begin{array}{l}\infty \\
\infty \\
\hat{N}\end{array}$ & 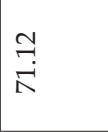 & $\begin{array}{l}\text { से } \\
\infty \\
\infty \\
0\end{array}$ \\
\hline & w & 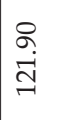 & $\begin{array}{l}0 \\
\infty \\
\infty \\
\infty \\
=\end{array}$ & İ & 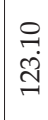 & 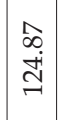 & $\begin{array}{l}0 \\
\hat{0} \\
\stackrel{1}{1}\end{array}$ & $\begin{array}{l}\infty \\
0 \\
0 \\
0 \\
\end{array}$ & స్ & $\frac{\mathfrak{\alpha}}{\mathfrak{a}}$ & 茫 & 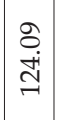 & 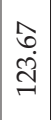 & స్ & $\begin{array}{l}\overrightarrow{\mathrm{N}} \\
\text { ลี }\end{array}$ & $\begin{array}{l}\stackrel{\circ}{\circ} \\
\stackrel{\circ}{\rho} \\
=\end{array}$ & $\stackrel{\infty}{\stackrel{\infty}{\sim}}$ \\
\hline \multirow[b]{4}{*}{$\begin{array}{l}\vec{z} \\
0 \\
0 \\
\dot{0} \\
0\end{array}$} & 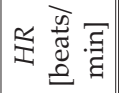 & $\begin{array}{l}8 \\
\infty \\
\infty\end{array}$ & $\begin{array}{l}8 \\
\stackrel{8}{8}\end{array}$ & $\begin{array}{l}8 \\
\text { مे }\end{array}$ & $\begin{array}{l}8 \\
\infty \\
\infty\end{array}$ & $\begin{array}{l}\infty \\
\text { ๙̇ } \\
\text { a }\end{array}$ & $\begin{array}{l}\text { P } \\
\text { ti }\end{array}$ & $\begin{array}{l}\text { స్ } \\
\text { ๙ू }\end{array}$ & \begin{tabular}{l}
8 \\
\multirow{0}{*}{}
\end{tabular} & तิ & $\begin{array}{l}\infty \\
\infty \\
\infty \\
\infty\end{array}$ & $\begin{array}{c}8 \\
\infty \\
\infty \\
\infty\end{array}$ & $\begin{array}{l}8 \\
1 \\
1\end{array}$ & 吕 & \begin{tabular}{l}
9 \\
\multirow{1}{0}{} \\
$\infty$
\end{tabular} & $\begin{array}{l}8 \\
\infty \\
\infty \\
\infty\end{array}$ & $\begin{array}{l}\text { సิ } \\
\text { ఎ }\end{array}$ \\
\hline & $\theta \frac{\bar{n}}{\theta}$ & ర్ & $\hat{\sigma}$ & : & : & $\begin{array}{l}0 \\
0 \\
0\end{array}$ & : & 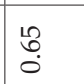 & in & 号 & 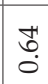 & $\begin{array}{l}0 \\
\stackrel{0}{0}\end{array}$ & $\begin{array}{l}0 \\
0 \\
0\end{array}$ & $\begin{array}{l}\infty \\
0 \\
0 \\
0\end{array}$ & $\begin{array}{l}\infty \\
0 \\
0 \\
0\end{array}$ & 官 & $\begin{array}{l}\sigma \\
0 \\
0\end{array}$ \\
\hline & 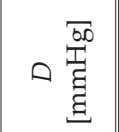 & $\begin{array}{l}8 \\
1 \\
1\end{array}$ & $\begin{array}{l}8 \\
0 \\
\infty \\
\infty\end{array}$ & $\begin{array}{l}8 \\
\dot{\infty} \\
\infty\end{array}$ & $\begin{array}{l}\text { तิ } \\
\text { i }\end{array}$ & $\begin{array}{l}8 \\
\dot{8} \\
+ \\
+\end{array}$ & 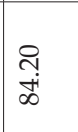 & $\begin{array}{l}\not{1} \\
10 \\
\infty\end{array}$ & $\begin{array}{l}8 \\
\dot{\infty} \\
\dot{\infty}\end{array}$ & $\begin{array}{l}8 \\
0 \\
\infty \\
\infty\end{array}$ & $\begin{array}{l}\infty \\
\infty \\
\infty \\
\infty\end{array}$ & $\begin{array}{l}\text { 유 } \\
\infty \\
\infty\end{array}$ & $\begin{array}{c}0 \\
+ \\
\infty \\
\infty \\
\infty\end{array}$ & $\begin{array}{l}\text { กิ } \\
\text { ฌే }\end{array}$ & $\begin{array}{l}8 \\
10 \\
\infty\end{array}$ & స઼. & $\begin{array}{l}8 \\
\text { i }\end{array}$ \\
\hline & 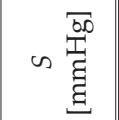 & 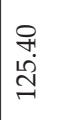 & $\begin{array}{l}\infty \\
\infty \\
\stackrel{0}{=}\end{array}$ & 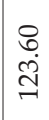 & 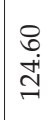 & 웜 & 空 & 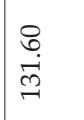 & $\stackrel{i}{+}$ & 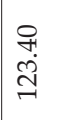 & $\begin{array}{l}\text { P+1 } \\
\stackrel{0}{0} \\
\stackrel{0}{-1}\end{array}$ & 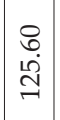 & $\begin{array}{l}\infty \\
\stackrel{1}{+} \\
\stackrel{+}{J}\end{array}$ & 足 & $\begin{array}{l}\stackrel{N}{1} \\
\stackrel{+}{+} \\
\rightarrow\end{array}$ & $\begin{array}{l}8 \\
\stackrel{1}{1} \\
1\end{array}$ & $\begin{array}{l}8 \\
0 \\
\infty \\
=\end{array}$ \\
\hline \multirow{3}{*}{ 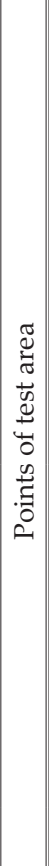 } & 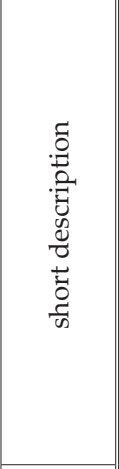 & 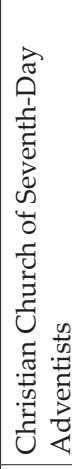 & 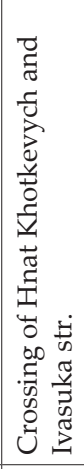 & 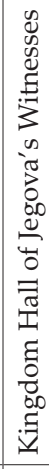 & 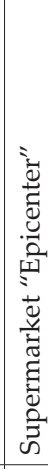 & 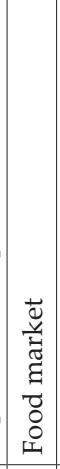 & 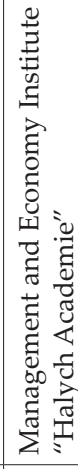 & 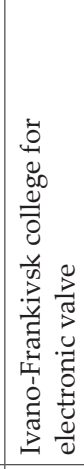 & 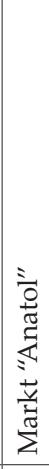 & 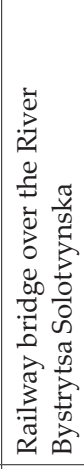 & 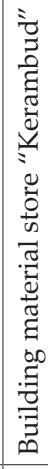 & 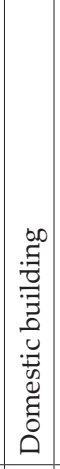 & 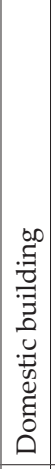 & 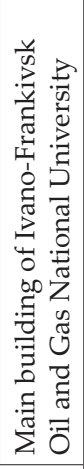 & 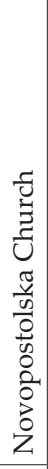 & 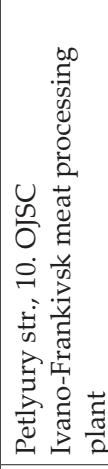 & 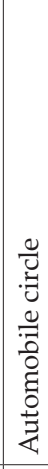 \\
\hline & 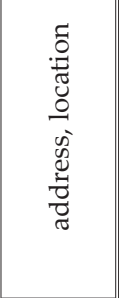 & 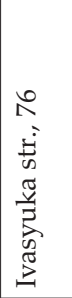 & 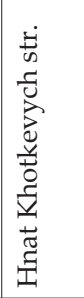 & 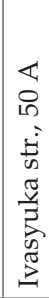 & 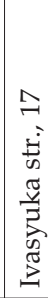 & $\begin{array}{l}\dot{ \pm} \\
\infty \\
0 \\
\tilde{D} \\
\omega\end{array}$ & 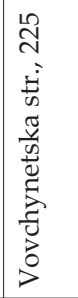 & 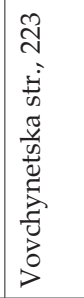 & 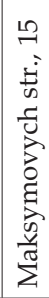 & 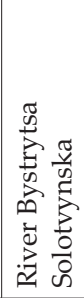 & 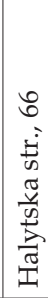 & 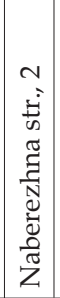 & 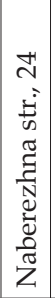 & 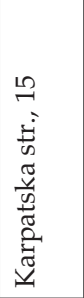 & 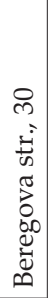 & 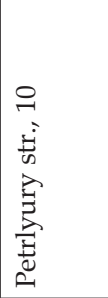 & $\begin{array}{l}0 \\
0 \\
0 \\
\dot{1} \\
0 \\
\frac{1}{0} \\
0 \\
0 \\
0 \\
0 \\
0 \\
0 \\
0 \\
0 \\
0\end{array}$ \\
\hline & $\dot{z}$ & f & F & $\stackrel{\infty}{\leftrightarrow}$ & 아 & ட̊ & $\overrightarrow{\text { ம }}$ & กิ & กิ & मे & L & เீ & in & $\infty)^{\infty}$ & เి & 8 & $\overrightarrow{6}$ \\
\hline
\end{tabular}




\begin{tabular}{|c|c|c|c|c|c|c|c|c|c|c|c|c|c|c|c|c|c|}
\hline $\begin{array}{l}\infty \\
\text { î } \\
\end{array}$ & $\begin{array}{l}\infty \\
\stackrel{0}{0} \\
0\end{array}$ & 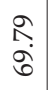 & $\begin{array}{l}\infty \\
\stackrel{0}{0} \\
+\end{array}$ & $\begin{array}{l}0 \\
\tilde{N} \\
i \\
\text { ñ }\end{array}$ & $\begin{array}{c}0 \\
i n \\
i \\
i n\end{array}$ & $\begin{array}{l}8 \\
\stackrel{+}{10}\end{array}$ & $\begin{array}{l}\infty \\
\infty \\
\infty \\
0\end{array}$ & $\begin{array}{l}\infty \\
\stackrel{\leftrightarrow}{\sigma} \\
\dot{b}\end{array}$ & $\begin{array}{l}\hat{b} \\
\text { in }\end{array}$ & $\begin{array}{l}0 \\
0 \\
0\end{array}$ & 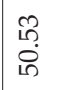 & $\begin{array}{l}\text { م. } \\
\text { กิ่ }\end{array}$ & 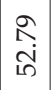 & 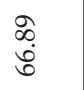 & $\begin{array}{l}\stackrel{0}{ } \\
\stackrel{+}{*}\end{array}$ & $\frac{7}{7}$ & $\begin{array}{l}\text { 울 } \\
\text { مि }\end{array}$ \\
\hline 莒 & $\stackrel{\stackrel{9}{+}}{\circ}$ & $\stackrel{8}{6}$ & 苚 & $\begin{array}{l}\stackrel{\leftrightarrow}{\circ} \\
\stackrel{\circ}{\circ}\end{array}$ & $\begin{array}{l}\infty \\
\stackrel{\infty}{0} \\
\stackrel{1}{0}\end{array}$ & ถึ. & $\begin{array}{l}8 \\
3 \\
0\end{array}$ & 苍 & 苜 & $\stackrel{8}{\circ}$ & 蒙 & గ్t & مึ & ட̊. & $\underset{\sigma}{\overrightarrow{0}}$ & 菅 & 占 \\
\hline $\begin{array}{l}\text { 엉 } \\
\text { î }\end{array}$ & $\begin{array}{l}\infty \\
\circ \\
\circ \\
\text { ம் }\end{array}$ & ָุ & $\stackrel{\wp}{0}$ & $\left|\begin{array}{c}0 \\
\stackrel{+}{+} \\
\dot{+}\end{array}\right|$ & 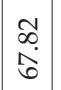 & 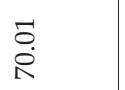 & $\begin{array}{c}0 \\
\infty \\
\infty \\
1\end{array}$ & $\begin{array}{l}8 \\
\infty \\
\dot{0} \\
\dot{0}\end{array}$ & 文 & $\underset{ }{8}$ & 옹 & $\frac{\sigma}{\sigma}$ & 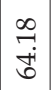 & 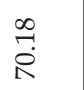 & $\underset{⿱ 亠 䒑}{\stackrel{\rightleftarrows}{*}}$ & \& & $\begin{array}{l}\infty \\
\stackrel{\sigma}{0} \\
\text { d }\end{array}$ \\
\hline $\begin{array}{l}\vec{\infty} \\
\stackrel{+}{\exists} \\
\vec{\exists}\end{array}$ & 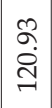 & 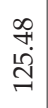 & 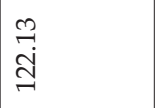 & 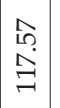 & $\begin{array}{l}\hat{A} \\
\stackrel{S}{Z} \\
\end{array}$ & $\begin{array}{l}\stackrel{\varnothing}{0} \\
\infty \\
\Rightarrow \\
\Rightarrow\end{array}$ & 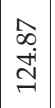 & $\begin{array}{l}\text { సે } \\
\text { ڤ } \\
\ddots\end{array}$ & 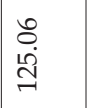 & 胥 & $\begin{array}{l}\infty \\
\stackrel{0}{6} \\
ٍ\end{array}$ & $\mid \begin{array}{c}0 \\
0 \\
\infty \\
\infty \\
=\end{array}$ & 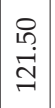 & $\begin{array}{l}\text { جे } \\
\stackrel{ }{\mathrm{I}}\end{array}$ & 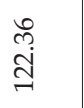 & 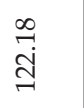 & 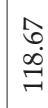 \\
\hline $\begin{array}{l}\stackrel{+}{+} \\
\infty \\
\infty\end{array}$ & \begin{tabular}{l}
8 \\
\hdashline \\
$\infty$ \\
$\infty$
\end{tabular} & $\begin{array}{l}\infty \\
\infty \\
\text { ๙่ }\end{array}$ & $\begin{array}{l}\stackrel{1}{1} \\
\infty \\
\infty\end{array}$ & $\begin{array}{l}8 \\
8 \\
8\end{array}$ & $\begin{array}{l}\infty \\
8 \\
8 \\
0\end{array}$ & 今े & $\begin{array}{c}\text { 尺े } \\
\text { ूू }\end{array}$ & $\begin{array}{l}8 \\
\text { ๙̇ }\end{array}$ & 움 & $\begin{array}{l}\text { ণิ } \\
\text { ฉి }\end{array}$ & ণิ & $\begin{array}{l}\infty \\
\infty \\
\end{array}$ & 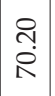 & $\begin{array}{l}\text { ণิ } \\
\text { ฉ̊ }\end{array}$ & $\begin{array}{l}8 \\
\text { i }\end{array}$ & ণ্ণ & $\stackrel{8}{\stackrel{1}{ }}$ \\
\hline $\begin{array}{l}\widetilde{O} \\
\stackrel{0}{\circ}\end{array}$ & 艿 & $\begin{array}{l}\infty \\
0 \\
0\end{array}$ & 苛 & $\begin{array}{c}0 \\
0 \\
0 \\
0 \\
0\end{array}$ & 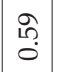 & $\stackrel{8}{0}$ & 它 & $\stackrel{+}{0}$ & ถึ. & $\stackrel{8}{\circ}$ & مُ & $\mid \begin{array}{c}\infty \\
\stackrel{\infty}{0} \\
0\end{array}$ & $\begin{array}{l}\mathrm{T} \\
\mathrm{S} \\
0\end{array}$ & ర్d & ণ్ర & ర్ & $\stackrel{\vec{b}}{0}$ \\
\hline ત્તે & ஓ & 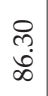 & 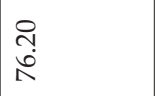 & $\begin{array}{l}8 \\
8 \\
\dot{1}\end{array}$ & 윰 & $\begin{array}{l}\text { 웃 } \\
\text { i }\end{array}$ & 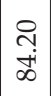 & $\begin{array}{l}\stackrel{9}{\sharp} \\
\stackrel{2}{R}\end{array}$ & 尺ે & $\begin{array}{c}\text { ָิ } \\
\text { ஸे }\end{array}$ & ำ & $\begin{array}{l}\text { กิ } \\
\stackrel{1}{1}\end{array}$ & 官 & 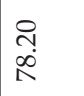 & $\begin{array}{l}\stackrel{2}{2} \\
\infty \\
\infty\end{array}$ & \& & $\begin{array}{l}\text { 공 } \\
\text { N }\end{array}$ \\
\hline 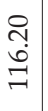 & 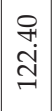 & \begin{tabular}{l} 
\& \\
సิ \\
\multirow{-}{*}{}
\end{tabular} & \begin{tabular}{l} 
ㄱ \\
ㅁ \\
\multirow{1}{*}{}
\end{tabular} & $\begin{array}{l}8 \\
\dot{a} \\
=\end{array}$ & $\begin{array}{l}\stackrel{1}{2} \\
\stackrel{2}{\exists}\end{array}$ & 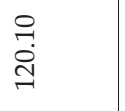 & 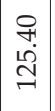 & 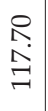 & 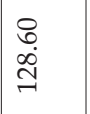 & $\begin{array}{l}8 \\
\stackrel{+}{J}\end{array}$ & $\begin{array}{l}\stackrel{0}{1} \\
\infty \\
\stackrel{0}{=}\end{array}$ & 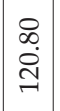 & 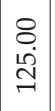 & 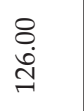 & 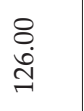 & $\begin{array}{l}8 \\
\stackrel{+}{\beth}\end{array}$ & $\begin{array}{l}\stackrel{\circ}{\circ} \\
\stackrel{2}{=}\end{array}$ \\
\hline 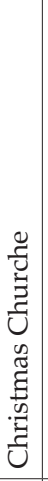 & 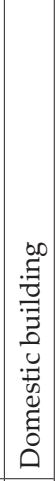 & $\begin{array}{l}\vec{y} \\
\vec{u} \\
\vec{v} \\
\vec{u} \\
\tilde{u}\end{array}$ & 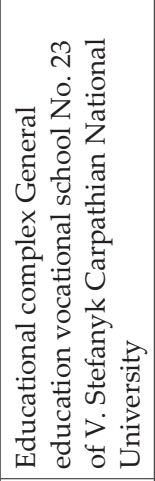 & 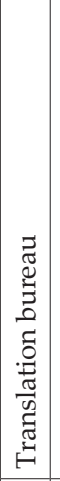 & 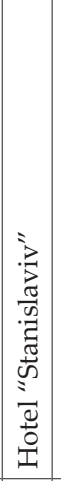 & 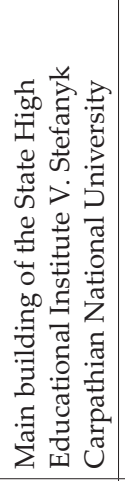 & 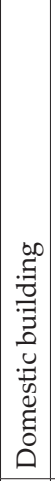 & 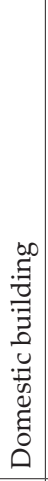 & 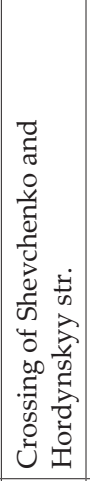 & 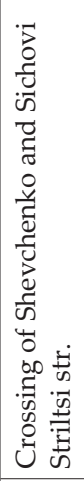 & 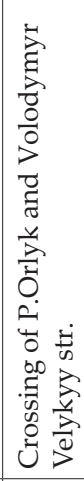 & 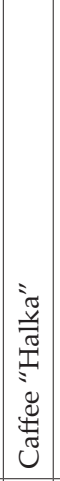 & 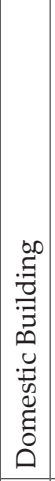 & 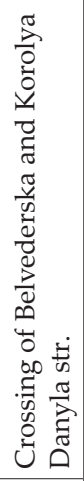 & 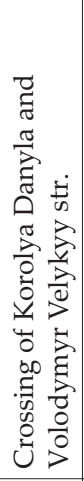 & 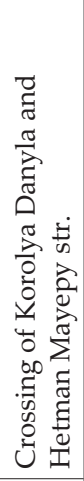 & 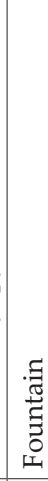 \\
\hline 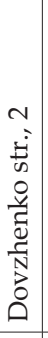 & 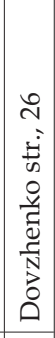 & 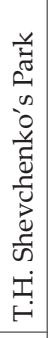 & 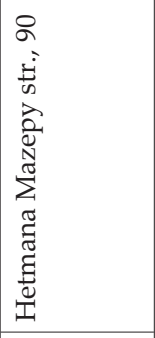 & 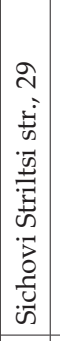 & 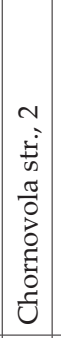 & 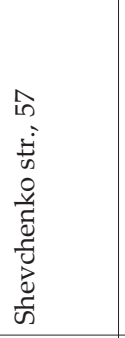 & 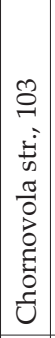 & 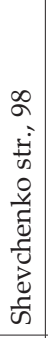 & 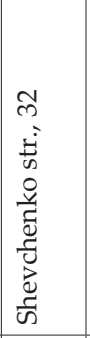 & 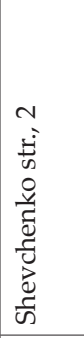 & 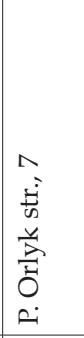 & 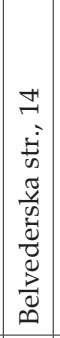 & 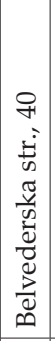 & 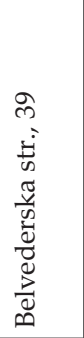 & 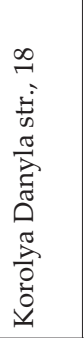 & 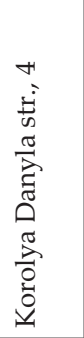 & 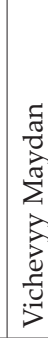 \\
\hline$\widetilde{\sigma}$ & 8 & تु & 18 & $\bullet$ & $\widehat{\theta}$ & $\infty$ & 8 & $尺$ & 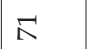 & $\mathbb{N}$ & $\Re$ & ホ & 늣 & $\stackrel{0}{2}$ & $\lesssim$ & $\stackrel{\infty}{\ltimes}$ & হ \\
\hline
\end{tabular}




\begin{tabular}{|c|c|c|c|c|c|c|c|c|c|c|c|c|c|c|c|c|c|c|c|}
\hline \multirow{4}{*}{ 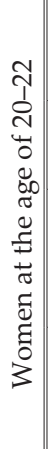 } & 离 & $\begin{array}{l}\infty \\
\infty \\
i n \\
i n\end{array}$ & $\begin{array}{l}\text { के } \\
\text { हnं }\end{array}$ & $\underset{\infty}{\vec{\infty}}$ & त्र & $\begin{array}{l}5 \\
\stackrel{5}{ } \\
\text { மn }\end{array}$ & $\begin{array}{l}\text { กิ } \\
\text { ลิ }\end{array}$ & $\stackrel{\circ}{\circ}$ & $\begin{array}{l}\stackrel{L}{2} \\
\text { ֶ̊ }\end{array}$ & 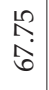 & $\stackrel{m}{\stackrel{0}{6}}$ & กิ & $\begin{array}{l}8 \\
\text { மं }\end{array}$ & $\begin{array}{l}\stackrel{8}{e} \\
\stackrel{i}{N}\end{array}$ & 굴 & $\begin{array}{l}\hat{0} \\
10 \\
10\end{array}$ & $\begin{array}{l}7 \\
8 \\
8\end{array}$ & $\begin{array}{l}10 \\
100 \\
10\end{array}$ & $\begin{array}{l}\infty \\
\stackrel{\infty}{+} \\
\text { + }\end{array}$ \\
\hline & $\theta \frac{\bar{\sigma}}{\theta}$ & $\stackrel{\substack{\infty \\
\hdashline \\
\hdashline}}{0}$ & 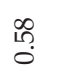 & హర & L̊. & กิ & $\begin{array}{l}\infty \\
\stackrel{\infty}{\infty} \\
0\end{array}$ & $\stackrel{8}{\circ}$ & $\begin{array}{l}\mathfrak{T} \\
0\end{array}$ & $\begin{array}{l}\text { L̊ } \\
0\end{array}$ & $\begin{array}{l}\infty \\
\stackrel{2}{0} \\
0\end{array}$ & $\begin{array}{l}\mathcal{T} \\
0 \\
0\end{array}$ & L̊ & 苞 & 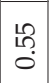 & 命 & مึ & 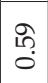 & $\begin{array}{l}\infty \\
\stackrel{1}{0} \\
0\end{array}$ \\
\hline & 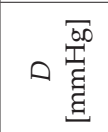 & 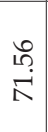 & $\stackrel{\hat{N}}{\Sigma}$ & $\begin{array}{l}\infty \\
0 \\
2 \\
2\end{array}$ & $\stackrel{1}{\overparen{2}}$ & $\begin{array}{l}\infty \\
\infty \\
0 \\
0\end{array}$ & 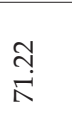 & $\begin{array}{l}\infty \\
\infty \\
0 \\
0\end{array}$ & $\begin{array}{l}\text { @̊ } \\
\stackrel{1}{N}\end{array}$ & $\begin{array}{l}\infty \\
\infty \\
\infty \\
0\end{array}$ & त̃ & 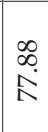 & مُ & 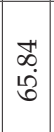 & \begin{tabular}{l}
$\infty$ \\
\multirow{1}{1}{} \\
10
\end{tabular} & $\begin{array}{l}0 \\
0 \\
0 \\
0 \\
0\end{array}$ & $\begin{array}{l}\infty \\
\stackrel{2}{\llcorner} \\
\stackrel{R}{R}\end{array}$ & $\frac{\tilde{\sigma}}{\dot{x}}$ & 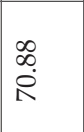 \\
\hline & 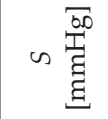 & $\begin{array}{l}\vec{n} \\
\underset{\mathrm{N}}{\mathrm{N}}\end{array}$ & $\begin{array}{l}8 \\
\text { ते } \\
\text { 1 }\end{array}$ & 골 & సે & $\begin{array}{l}\infty \\
o \\
\sigma \\
= \\
=\end{array}$ & 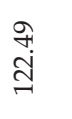 & $\begin{array}{l}8 \\
\stackrel{0}{\exists} \\
\end{array}$ & 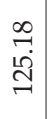 & 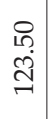 & $\frac{\sqrt{n}}{\stackrel{N}{N}}$ & 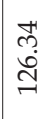 & 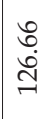 & 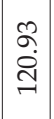 & 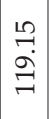 & $\begin{array}{l}\infty \\
\infty \\
\stackrel{1}{=} \\
=\end{array}$ & 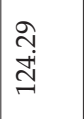 & 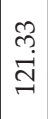 & ลิ \\
\hline \multirow{4}{*}{ 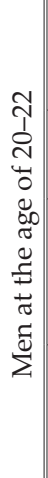 } & 离 & $\begin{array}{l}8 \\
\stackrel{i}{1}\end{array}$ & $\begin{array}{l}\infty \\
\infty \\
1\end{array}$ & $\begin{array}{l}0 \\
\text { s. } \\
\text { oे }\end{array}$ & $\begin{array}{l}8 \\
\dot{1}\end{array}$ & $\begin{array}{l}8 \\
\infty \\
\infty\end{array}$ & $\begin{array}{l}\text { ํ } \\
\text { gi }\end{array}$ & $\begin{array}{l}\text { ते } \\
\text { ळ }\end{array}$ & $\begin{array}{l}\stackrel{8}{ } \\
\text { बं }\end{array}$ & $\begin{array}{l}8 \\
\text { б̆ }\end{array}$ & $\begin{array}{l}\text { กิ } \\
\text { ฉू }\end{array}$ & กุ & $\begin{array}{l}8 \\
\stackrel{+}{1}\end{array}$ & \begin{tabular}{|c|}
$\infty$ \\
$\infty$ \\
$\alpha$ \\
$\sigma$ \\
\end{tabular} & $\begin{array}{l}8 \\
\dot{b} \\
\infty \\
\infty\end{array}$ & $\begin{array}{c}\text { त्र } \\
\infty \\
\infty \\
\infty\end{array}$ & $\begin{array}{l}\text { P } \\
+ \\
\text { க }\end{array}$ & $\begin{array}{c}8 \\
\infty \\
\infty \\
\infty\end{array}$ & $\begin{array}{c}8 \\
\dot{0} \\
\infty\end{array}$ \\
\hline & $\ominus \frac{\pi}{\varrho}$ & $\vec{b}$ & 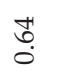 & $\begin{array}{l}\infty \\
0 \\
0\end{array}$ & 苛 & 䘾 & i़े & 苞 & $\begin{array}{l}\vec{\sigma} \\
0\end{array}$ & $\begin{array}{l}\vec{\sigma} \\
0\end{array}$ & $\begin{array}{l}\tilde{O} \\
0\end{array}$ & ț & $\vec{b}$ & $\begin{array}{l}\hat{0} \\
0\end{array}$ & $\begin{array}{l}\tilde{S} \\
0 \\
0\end{array}$ & $\begin{array}{l}3 \\
0 \\
0\end{array}$ & $\vec{b}$ & $\begin{array}{l}10 \\
0 \\
0\end{array}$ & $\begin{array}{l}3 \\
0 \\
0\end{array}$ \\
\hline & 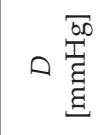 & $\begin{array}{l}\text { กิ } \\
\text { م }\end{array}$ & \begin{tabular}{l}
9 \\
\multirow{2}{*}{} \\
$\infty$
\end{tabular} & $\begin{array}{l}\infty \\
\infty \\
\infty \\
\infty\end{array}$ & 导 & तึ & $\begin{array}{l}\text { तิ } \\
\text { ホ̦ }\end{array}$ & $\begin{array}{l}\text { సิ } \\
\text { है }\end{array}$ & $\begin{array}{l}\stackrel{8}{\infty} \\
\stackrel{1}{N}\end{array}$ & 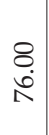 & $\begin{array}{l}8 \\
\infty \\
0 \\
n\end{array}$ & $\begin{array}{c}8 \\
i \\
\infty \\
\infty\end{array}$ & $\begin{array}{l}\text { సิ } \\
\infty \\
\infty\end{array}$ & \begin{tabular}{|c|} 
\\
0 \\
$\dot{\infty}$ \\
\end{tabular} & $\begin{array}{c}8 \\
10 \\
1\end{array}$ & $\begin{array}{l}8 \\
0 \\
0 \\
1\end{array}$ & م̊ & 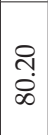 & $\begin{array}{c}8 \\
\infty \\
\infty \\
10\end{array}$ \\
\hline & 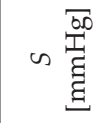 & 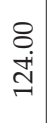 & $\begin{array}{l}\text { 움 } \\
\stackrel{1}{2} \\
i\end{array}$ & 옴 & 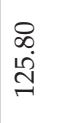 & $\begin{array}{l}9 \\
9 \\
\infty \\
= \\
7\end{array}$ & 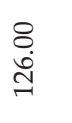 & 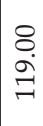 & $\begin{array}{l}\text { \& } \\
\text { Ầ } \\
\text { - }\end{array}$ & $\begin{array}{l}8 \\
\stackrel{8}{1} \\
\stackrel{+}{-}\end{array}$ & 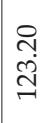 & 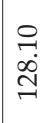 & 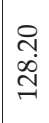 & $\begin{array}{l}\text { P } \\
\text { i } \\
\text { }\end{array}$ & 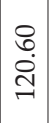 & 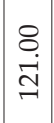 & \begin{tabular}{l}
$\infty$ \\
$\infty$ \\
$\stackrel{1}{1}$ \\
\multirow{1}{1}{}
\end{tabular} & 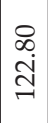 & ָี \\
\hline \multirow{3}{*}{ 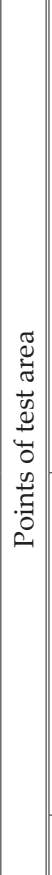 } & 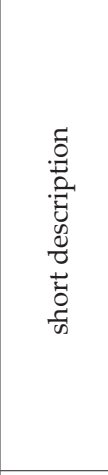 & 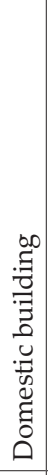 & 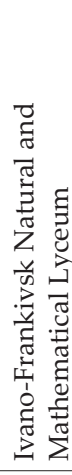 & 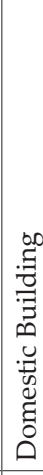 & 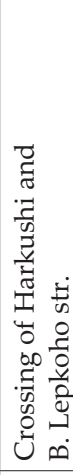 & 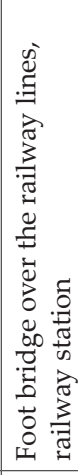 & 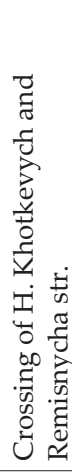 & 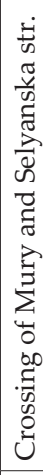 & 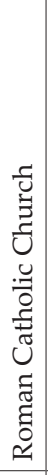 & 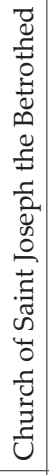 & 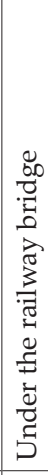 & 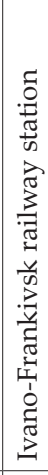 & 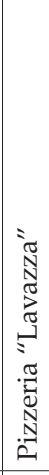 & 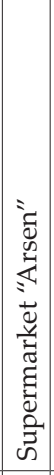 & 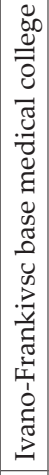 & 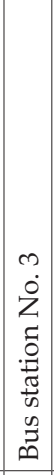 & 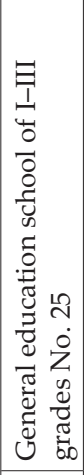 & 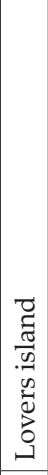 & 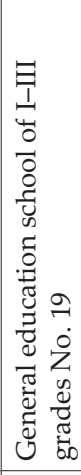 \\
\hline & 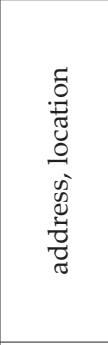 & 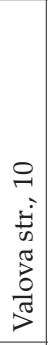 & 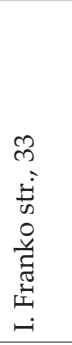 & 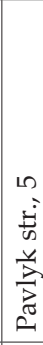 & 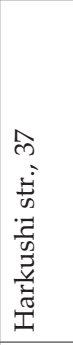 & 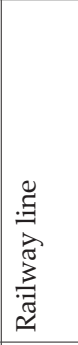 & 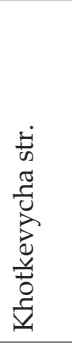 & B & 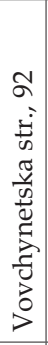 & 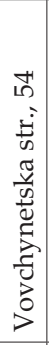 & 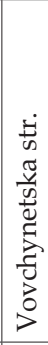 & 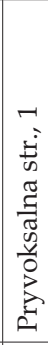 & 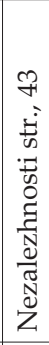 & 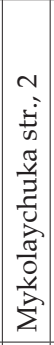 & 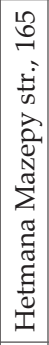 & 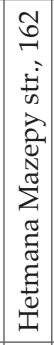 & 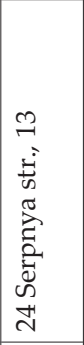 & 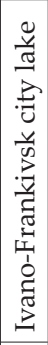 & 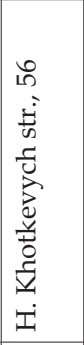 \\
\hline & 2 & $\infty$ & $\infty$ & $\infty$ & $\infty$ & \$ & $\infty$ & $\infty$ & $\hat{\infty}$ & $\begin{array}{l}\infty \\
\infty\end{array}$ & $\infty$ & ஓ & $\bar{\sigma}$ & สু & $\alpha$ & J゙ & 으 & ๙ & 人̆ \\
\hline
\end{tabular}




\begin{tabular}{|c|c|c|c|c|c|c|c|c|c|c|c|c|c|c|}
\hline $\begin{array}{l}\text { भે } \\
\text { ம் }\end{array}$ & $\begin{array}{l}\stackrel{3}{7} \\
\infty \\
i n\end{array}$ & 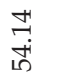 & $\begin{array}{l}\text { సે } \\
\text { மn }\end{array}$ & $\begin{array}{l}20 \\
0 \\
0 \\
0\end{array}$ & $\begin{array}{l}\overrightarrow{0} \\
0 \\
0 \\
0\end{array}$ & $\begin{array}{l}\sigma \\
\hat{\sigma}\end{array}$ & $\begin{array}{l}\text { तై } \\
\text { రి }\end{array}$ & $\begin{array}{l}\text { तิ } \\
\text { in } \\
\text { in }\end{array}$ & $\begin{array}{l}\stackrel{\infty}{\stackrel{0}{0}} \\
\stackrel{8}{0}\end{array}$ & लि. & 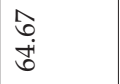 & $\begin{array}{l}\dot{j} \\
g \\
g\end{array}$ & $\begin{array}{l}3 \\
\infty \\
\infty \\
\infty\end{array}$ & $\begin{array}{l}\hat{6} \\
\text { ம் }\end{array}$ \\
\hline $\begin{array}{l}-\overrightarrow{6} \\
\stackrel{0}{0}\end{array}$ & 芯 & 命 & గิ గุ & กิ & $\begin{array}{c}\infty \\
i \\
0 \\
0\end{array}$ & 命 & ర్ర & ث艹 & $\stackrel{\circ}{8}$ & $\stackrel{\text { Ln }}{\stackrel{L}{0}}$ & $\begin{array}{c}\infty \\
\substack{10 \\
0}\end{array}$ & டั? & 命 & 茴 \\
\hline 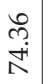 & $\begin{array}{l}\ddot{8} \\
\stackrel{0}{\circ}\end{array}$ & 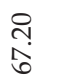 & $\begin{array}{l}\text { 足 } \\
\stackrel{0}{0}\end{array}$ & $\begin{array}{l}8 \\
\dot{3}\end{array}$ & $\stackrel{\wp}{\dot{1}}$ & $\begin{array}{c}\hat{\alpha} \\
\infty \\
0\end{array}$ & $\underset{\sim}{\stackrel{\infty}{\infty}}$ & $\begin{array}{l}\overrightarrow{\mid r n} \\
\stackrel{+}{6}\end{array}$ & $\begin{array}{l}\stackrel{L}{\llcorner} \\
\stackrel{N}{N}\end{array}$ & $\begin{array}{l}\text { त् } \\
\text { تु }\end{array}$ & $\begin{array}{l}\infty \\
0 \\
0\end{array}$ & $\stackrel{2}{\stackrel{1}{n}}$ & $\stackrel{\overrightarrow{0}}{\stackrel{0}{0}}$ & $\stackrel{M}{\stackrel{9}{\pi}}$ \\
\hline 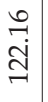 & $\begin{array}{l}\infty \\
\stackrel{\infty}{\sigma} \\
\sigma \\
=\end{array}$ & $\begin{array}{l}\stackrel{0}{ } \\
\sim \\
\infty \\
\rightleftharpoons\end{array}$ & $\begin{array}{l}8 \\
\stackrel{0}{\circ} \\
\exists\end{array}$ & în & $\begin{array}{l}\stackrel{ }{1} \\
\stackrel{\vec{\jmath}}{\mathbb{Z}}\end{array}$ & 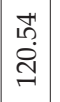 & 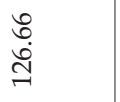 & $\begin{array}{l}\stackrel{0}{\wedge} \\
\infty \\
ٍ\end{array}$ & $\begin{array}{l}\stackrel{M}{ت} \\
\stackrel{-}{二}\end{array}$ & $\begin{array}{l}8 \\
\stackrel{8}{0} \\
=\end{array}$ & 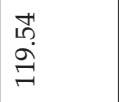 & $\begin{array}{l}\text { ○̊ } \\
\text { సิ }\end{array}$ & 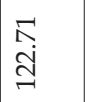 & 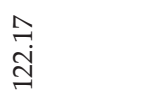 \\
\hline $\begin{array}{l}8 \\
\qquad \\
\qquad\end{array}$ & $\begin{array}{l}8 \\
\infty \\
\infty \\
\infty\end{array}$ & $\begin{array}{l}8 \\
\stackrel{i}{ }\end{array}$ & $\begin{array}{l}8 \\
\stackrel{2}{1}\end{array}$ & $\begin{array}{l}\text { P! } \\
\text { \& }\end{array}$ & 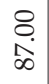 & $\begin{array}{l}8 \\
8 \\
8\end{array}$ & $\begin{array}{l}\infty \\
\infty \\
\infty \\
\infty\end{array}$ & $\begin{array}{l}\stackrel{9}{+} \\
\stackrel{1}{N}\end{array}$ & $\begin{array}{l}8 \\
\infty \\
\infty \\
\infty\end{array}$ & $\begin{array}{l}\stackrel{1}{1} \\
\infty \\
\infty \\
0\end{array}$ & $\begin{array}{l}8 \\
\infty \\
\infty\end{array}$ & $\begin{array}{l}\text { ָิ } \\
\text { ूু }\end{array}$ & $\begin{array}{l}\text { तે } \\
\text { ฉి }\end{array}$ & $\begin{array}{l}\text { શิ } \\
\text { ํㅗㅅ }\end{array}$ \\
\hline $\begin{array}{l}\stackrel{0}{0} \\
\stackrel{0}{0}\end{array}$ & 它 & $\begin{array}{l}\stackrel{0}{0} \\
\stackrel{0}{0}\end{array}$ & ڤึ. & $\begin{array}{l}\infty \\
\stackrel{\infty}{n} \\
0\end{array}$ & $\begin{array}{l}\mathscr{B} \\
\dot{0} \\
0\end{array}$ & $\begin{array}{l}\tilde{W} \\
0 \\
0\end{array} \mid$ & ت্ّ & $\begin{array}{l}\stackrel{0}{0} \\
\stackrel{0}{0}\end{array}$ & $\stackrel{10}{: 0}$ & ڤ़่ & 苂 & ڤ్. & 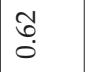 & $\begin{array}{l}\stackrel{8}{0} \\
\stackrel{0}{0}\end{array}$ \\
\hline $\begin{array}{c}8 \\
\text { i } \\
\infty\end{array}$ & 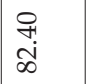 & 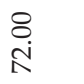 & $\begin{array}{l}8 \\
\stackrel{1}{0}\end{array}$ & $\begin{array}{l}8 \\
\text { i }\end{array}$ & $\begin{array}{c}8 \\
\infty \\
\infty \\
\infty\end{array}$ & $\begin{array}{l}8 \\
0 \\
1\end{array}$ & $\underset{\substack{\text { O } \\
\text { i }}}{ }$ & $\stackrel{\infty}{\stackrel{\infty}{\wedge}}$ & $\begin{array}{l}\text { ते } \\
\text { ڤิ }\end{array}$ & $\begin{array}{l}\text { กิ } \\
\text { হి }\end{array}$ & 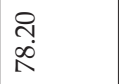 & 尔 & 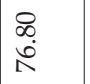 & $\begin{array}{l}\text { तิ } \\
\stackrel{+}{\leftarrow}\end{array}$ \\
\hline $\begin{array}{l}8 \\
\stackrel{+}{ \pm} \\
\underset{Z}{ }\end{array}$ & 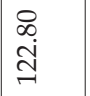 & $\begin{array}{l}8 \\
\stackrel{8}{0} \\
\text { ㄱ. }\end{array}$ & $\begin{array}{l}\stackrel{8}{0} \\
\stackrel{-}{\sigma}\end{array}$ & 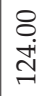 & 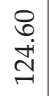 & $\begin{array}{l}8 \\
\dot{Z} \\
\mathbb{Z}\end{array}$ & 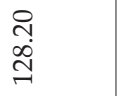 & 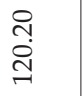 & 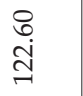 & $\begin{array}{l}8 \\
\infty \\
\infty \\
=\end{array}$ & 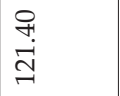 & 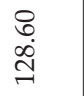 & $\begin{array}{l}\text { ণิ } \\
\stackrel{\text { I }}{\sim}\end{array}$ & $\begin{array}{l}\stackrel{8}{ } \\
\stackrel{+}{ \pm}\end{array}$ \\
\hline 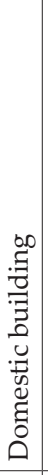 & 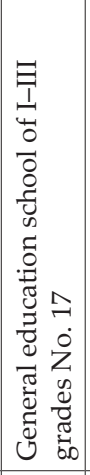 & 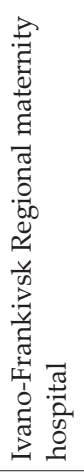 & 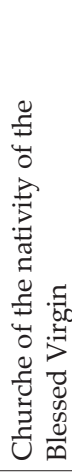 & 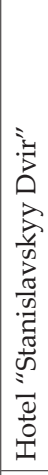 & 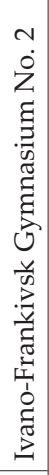 & 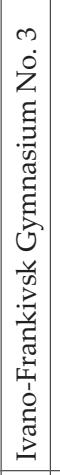 & 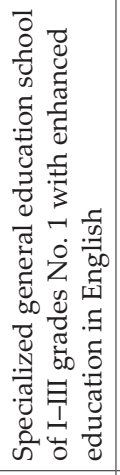 & 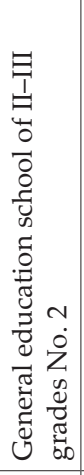 & 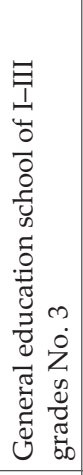 & 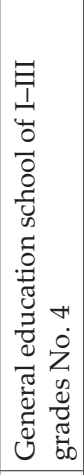 & 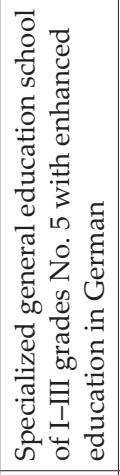 & 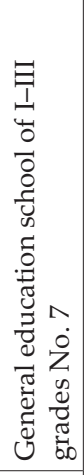 & 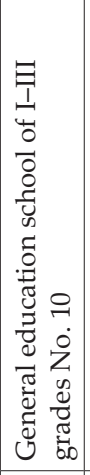 & 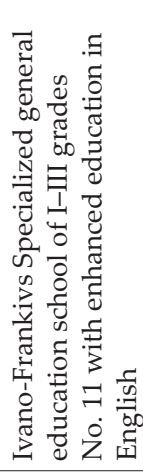 \\
\hline 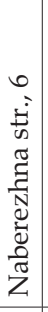 & 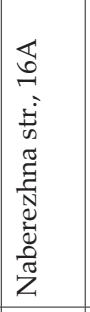 & 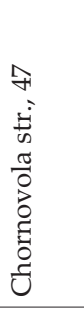 & $\begin{array}{l}\overleftrightarrow{3} \\
0 \\
\dot{1} \\
0 \\
\frac{\pi}{0} \\
0 \\
0 \\
0 \\
E \\
0 \\
d \\
\end{array}$ & 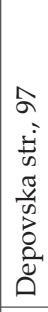 & 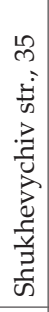 & 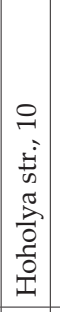 & 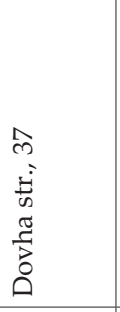 & 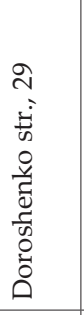 & 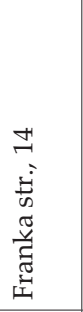 & 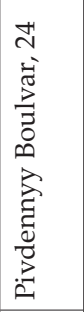 & 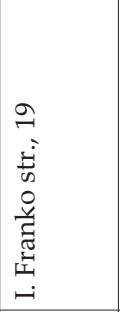 & 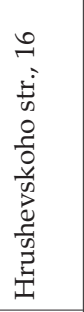 & 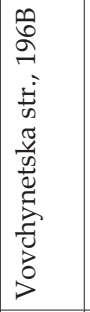 & 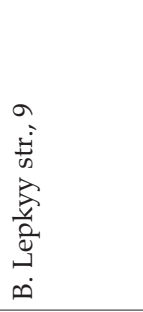 \\
\hline$\stackrel{\circ}{\circ}$ & ๙ & $\stackrel{8}{\circ}$ & 호 & $\stackrel{\sigma}{\sigma}$ & $\stackrel{3}{0}$ & 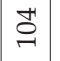 & 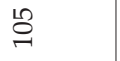 & $\stackrel{\overbrace{}}{\varrho}$ & $\stackrel{ }{\circ}$ & $\stackrel{\infty}{\stackrel{1}{\circ}}$ & g) & $\stackrel{ }{\leftrightarrows}$ & $\exists$ & $\stackrel{ }{\exists}$ \\
\hline
\end{tabular}




\begin{tabular}{|c|c|c|c|c|c|c|c|c|c|c|c|}
\hline \multirow{4}{*}{ 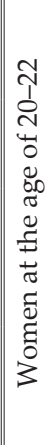 } & 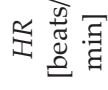 & $\begin{array}{l}\text { 员 } \\
8 \\
8\end{array}$ & $\begin{array}{l}8 \\
8 \\
0 \\
0\end{array}$ & 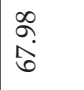 & 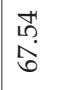 & $\frac{8}{6}$ & $\stackrel{m}{\overparen{6}}$ & हై & $\begin{array}{l}\stackrel{9}{7} \\
\infty \\
0\end{array}$ & $\begin{array}{l}\infty \\
0 \\
0\end{array}$ & $\begin{array}{l}\infty \\
\infty \\
\infty \\
10\end{array}$ \\
\hline & $\theta \frac{\bar{\sigma}}{\theta}$ & $\stackrel{\sigma}{\sigma}$ & مै & 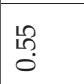 & $\begin{array}{l}0 \\
0 \\
0\end{array}$ & Sै & $\begin{array}{l}\infty \\
\stackrel{\infty}{0} \\
0\end{array}$ & B? & 苑 & ర్ & రై \\
\hline & $\curvearrowright{ }^{\varpi 00}$ & $\begin{array}{l}\stackrel{H}{N} \\
\infty \\
0\end{array}$ & $\begin{array}{l}\stackrel{1}{N} \\
\infty \\
\infty \\
0\end{array}$ & 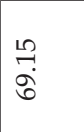 & $\begin{array}{l}\infty \\
\stackrel{\infty}{+} \\
\stackrel{+}{n}\end{array}$ & $\begin{array}{l}\infty \\
\text { in } \\
\text { in }\end{array}$ & 홍 & $\begin{array}{l}8 \\
\text { in } \\
\text { N }\end{array}$ & $\stackrel{8}{\circ}$ & $\begin{array}{l}\infty \\
ٌ \\
2 \\
\hat{R}\end{array}$ & $\begin{array}{l}\text { ते } \\
\text { నं }\end{array}$ \\
\hline & 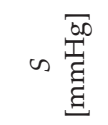 & $\begin{array}{l}\text { Bర } \\
\stackrel{\text { I }}{\exists}\end{array}$ & 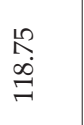 & 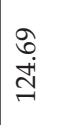 & $\begin{array}{l}\text { î } \\
\stackrel{m}{1} \\
\text { I }\end{array}$ & $\begin{array}{l}\infty \\
\stackrel{\infty}{0} \\
\stackrel{0}{\circ} \\
=\end{array}$ & Эี & $\begin{array}{l}\tilde{1} \\
\infty \\
\stackrel{2}{=} \\
=\end{array}$ & $\begin{array}{l}0 \\
m \\
\infty \\
0 \\
0\end{array}$ & $\begin{array}{l}\text { ָे } \\
\text { in } \\
=\end{array}$ & $\begin{array}{l}0 \\
\stackrel{1}{2} \\
\stackrel{1}{=}\end{array}$ \\
\hline \multirow{4}{*}{ 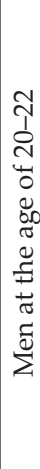 } & 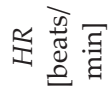 & $\begin{array}{l}\text { तิ } \\
\text { ஸे }\end{array}$ & $\begin{array}{l}8 \\
\infty \\
\infty \\
\infty\end{array}$ & $\begin{array}{l}\text { 9 } \\
\text { \&̊ }\end{array}$ & $\begin{array}{l}\text { तิ } \\
\text { ฉू }\end{array}$ & $\begin{array}{l}\text { O } \\
\text { i } \\
\infty\end{array}$ & 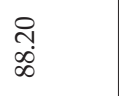 & 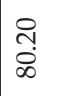 & ஓ̊ & \& & $\begin{array}{l}\text { त् } \\
\infty \\
\infty\end{array}$ \\
\hline & $\theta \frac{\bar{\sigma}}{\varrho}$ & $\stackrel{\text { :ூ }}{\circ}$ & 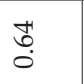 & $\stackrel{\vec{b}}{0}$ & $\stackrel{8}{0}$ & $\vec{b}$ & $\stackrel{8}{0}$ & 豖 & $\stackrel{\hat{0}}{0}$ & $\stackrel{8}{\circ}$ & $\stackrel{\hat{0}}{0}$ \\
\hline & 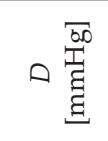 & $\begin{array}{l}\text { Iิ } \\
\stackrel{+}{N}\end{array}$ & 윰 & $\begin{array}{l}8 \\
1 \\
1\end{array}$ & $\begin{array}{l}9 \\
\text { i } \\
\text { i } \\
\infty\end{array}$ & P & $\begin{array}{l}\text { స్ } \\
\infty \\
\infty\end{array}$ & 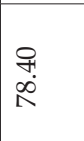 & $\begin{array}{l}\text { กิ } \\
\infty \\
\infty\end{array}$ & $\begin{array}{l}\text { N } \\
\infty \\
\infty\end{array}$ & $\begin{array}{l}8 \\
\stackrel{\infty}{\infty}\end{array}$ \\
\hline & 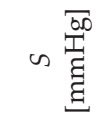 & $\begin{array}{l}8 \\
\stackrel{+}{+} \\
\exists\end{array}$ & 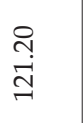 & $\begin{array}{l}\text { તิ } \\
\text { ปู } \\
\text { స్}\end{array}$ & 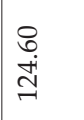 & $\begin{array}{l}8 \\
\infty \\
= \\
=\end{array}$ & $\underset{8}{\stackrel{8}{1}}$ & $\begin{array}{l}\text { సิ } \\
\text { மี } \\
=\end{array}$ & 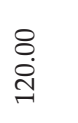 & $\begin{array}{l}8 \\
\stackrel{8}{2} \\
=\end{array}$ & $\begin{array}{l}8 \\
\stackrel{0}{0} \\
=\end{array}$ \\
\hline \multirow{3}{*}{ 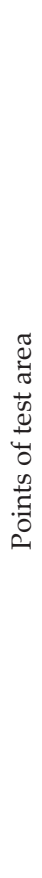 } & 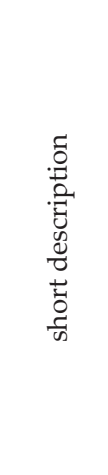 & 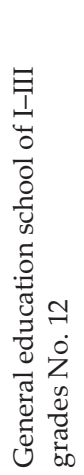 & 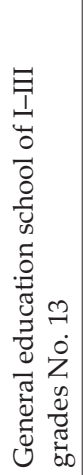 & 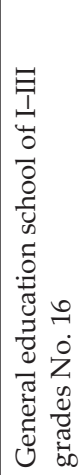 & 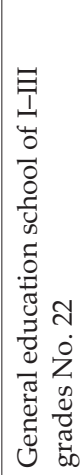 & 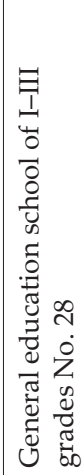 & 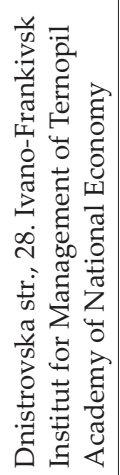 & 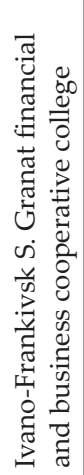 & 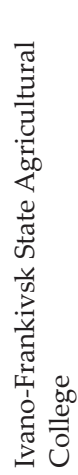 & 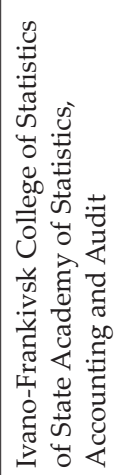 & 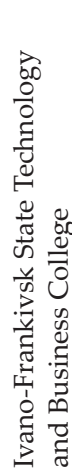 \\
\hline & 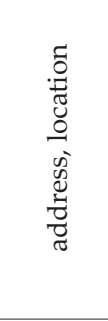 & 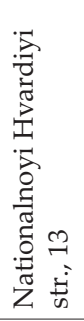 & 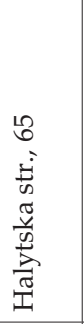 & 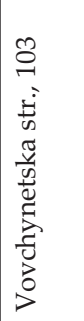 & 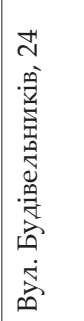 & 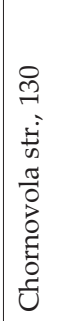 & 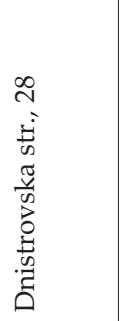 & 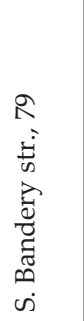 & 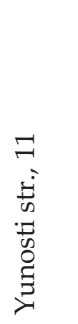 & 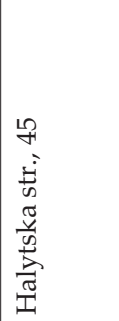 & 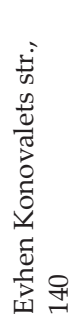 \\
\hline & $\dot{z}$ & $\stackrel{M}{=}$ & $\underset{\exists}{\nexists}$ & $\stackrel{10}{=}$ & $\stackrel{0}{=}$ & 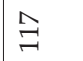 & $\stackrel{\infty}{=}$ & $\stackrel{\vartheta}{\rightleftharpoons}$ & તิ & $\vec{I}$ & ปี \\
\hline
\end{tabular}




\section{Methods of Statistical Research}

For the obtained data reproduced correlation coefficients were calculated using spreadsheet Microsoft Excel (CORREL functioned) for indicators $S, D, \varphi$ and heart rate (Tab. 2) and indicators $E_{\max ^{\prime}} H_{\max ^{\prime}} \mu_{\text {max }} E_{\text {aver }} H_{\text {aver }} \mu_{\text {aver }}$ (Tab. 1) separately for men and women. The results of the relevant calculations are represented in the Tables 3 and 4.

Table 3. The index of correlation between the value $S, D, \varphi, H R$ and $E_{\max ^{\prime}} H_{\max ^{\prime}} \mu_{\max ^{\prime}} E_{\text {aver }^{\prime}}$ $H_{\text {aver }} \mu_{\text {aver }}$ in the points of test area, the men at the age of $20-22$

\begin{tabular}{|l|c|c|c|c||}
\hline \multicolumn{1}{|c|}{ Parameters } & $S[\mathrm{mmHg}]$ & $D[\mathrm{mmHg}]$ & $\varphi[D / S]$ & $H R[$ beats $/ \mathrm{min}]$ \\
\hline \hline$E_{\max }[\mathrm{V} / \mathrm{m}]$ & -0.0068 & -0.3018 & -0.3487 & -0.3249 \\
\hline$H_{\max }[\mathrm{mA} / \mathrm{m}]$ & 0.0058 & -0.2813 & -0.3351 & -0.3260 \\
\hline$\mu_{\max }\left[\mathrm{mW} / \mathrm{cm}^{2}\right]$ & -0.0242 & -0.2524 & -0.2778 & -0.3824 \\
\hline$E_{\text {aver }}[\mathrm{V} / \mathrm{m}]$ & -0.0023 & -0.2951 & -0.3418 & -0.3767 \\
\hline$H_{\text {aver }}[\mathrm{mA} / \mathrm{m}]$ & 0.0014 & -0.2868 & -0.3375 & -0.3778 \\
\hline$\mu_{\text {aver }}\left[\mathrm{mW} / \mathrm{cm}^{2}\right]$ & -0.0820 & -0.3261 & -0.3186 & -0.3458 \\
\hline
\end{tabular}

Table 4. The index of correlation between the value $S, D, \varphi, H R$ and $E_{\max ^{\prime}} H_{\max ^{\prime}} \mu_{\max ^{\prime}} E_{\text {aver }^{\prime}}$ $H_{\text {aver }} \mu_{\text {aver }}$ in the points of test area in the points of test area, women at the age of 20-22

\begin{tabular}{||l|c|c|c|c||}
\hline \multicolumn{1}{|c|}{ Parameters } & $S[\mathrm{mmHg}]$ & $D[\mathrm{mmHg}]$ & $\varphi[D / S]$ & $H R$ [beats/min] \\
\hline \hline$E_{\max }[\mathrm{V} / \mathrm{m}]$ & 0.0033 & -0.2811 & -0.3254 & -0.2922 \\
\hline$H_{\max }[\mathrm{mA} / \mathrm{m}]$ & 0.0151 & -0.2622 & -0.3111 & -0.2951 \\
\hline$\mu_{\max }\left[\mathrm{mW} / \mathrm{cm}^{2}\right]$ & -0.0128 & -0.2631 & -0.2946 & -0.3315 \\
\hline$E_{\text {aver }}[\mathrm{V} / \mathrm{m}]$ & 0.0185 & -0.2449 & -0.2925 & -0.3101 \\
\hline$H_{\text {aver }}[\mathrm{mA} / \mathrm{m}]$ & 0.0248 & -0.2247 & -0.2744 & -0.4133 \\
\hline$\mu_{\text {aver }}\left[\mathrm{mW} / \mathrm{cm}^{2}\right]$ & -0.0624 & -0.2236 & -0.22033 & -0.3075 \\
\hline
\end{tabular}

The index of correlation $r$ can take the value in the interval from -1 to +1 , it means $-1 \leq r \leq 1$. The correlation relationship can be positive (direct) and negative (inverse). If $r>0$, then it talks about positive correlation or direct connection between values when with increasing of one value increases on the average value of the others. If $r<0$, the correlation is negative and it is inverse relationship between values. This means that during the growth of the second value will on the average decrease [9].

According to the data of correlation analysis is seen moderate negative correlation relationship between heart rate in men and women and measures $E_{\max ^{\prime}} H_{\text {max }^{\prime}}$ $\mu_{\text {max }} E_{\text {aver }} H_{\text {aver }}, \mu_{\text {aver }}$. Moreover, the maximum relationship is observed between heart rate in men and $\mu$ max. and heart rate in women and $H_{\text {aver }}$. 
The indexes of the correlation as a measure of relation between random values are also random values, and have stohestic nature. The index of correlation as sample characteristics, is tested for significance due to Student criterion $t$. The real value $t$ of the statistics $t_{\exp }$ is compared with the table value $t$-distribution with $n+m-2$ degrees of freedom, and by the given significant level $p<0.05$. If, $\left|t_{\exp }\right|>t_{\text {tabl }}$ it can be concluded that the index of the correlation is positiv (significant), and the relationship between the dependent variable and all independent factors is significant [11].

For the obtained data was calculated paired Student $t$ criterion with one sided distribution using spreadsheet Microsoft Excel (TTEST function) for the values S; D; $\varphi$ and heart rate (Tab. 3) and the values $E_{\max ^{\prime}} H_{\max ^{\prime}} \mu_{\max ^{\prime}} E_{\text {aver }^{\prime}} H_{\text {aver }^{\prime}} \mu_{\text {aver }}$ (Tab. 2) separately for men and women. The results of the relevant calculations are represented in Tables 5 and 6.

Table 5. Paired $t$-Student criterion with one sided distribution $\left(t_{\exp }\right)$, men at the age of 20-22

\begin{tabular}{||l|c|c|c|c||}
\hline \multicolumn{1}{|c|}{ Parameters } & $S[\mathrm{mmHg}]$ & $D[\mathrm{mmHg}]$ & $\varphi[D / S]$ & $H R[$ beats/min] \\
\hline$E_{\max }[\mathrm{V} / \mathrm{m}]$ & 3.271 & 4.745 & 8.425 & 1.263 \\
\hline$H_{\max }[\mathrm{mA} / \mathrm{m}]$ & 3.938 & 4.183 & 6.270 & 1.898 \\
\hline$\mu_{\max }\left[\mathrm{mW} / \mathrm{cm}^{2}\right]$ & 7.321 & 9.407 & 0.001 & 3.074 \\
\hline$E_{\text {aver }}[\mathrm{V} / \mathrm{m}]$ & 2.843 & 1.208 & 0.001 & 7.891 \\
\hline$H_{\text {aver }}[\mathrm{mA} / \mathrm{m}]$ & 5.385 & 4.369 & 8.143 & 2.220 \\
\hline$\mu_{\text {aver }}\left[\mathrm{mW} / \mathrm{cm}^{2}\right]$ & 4.093 & 2.585 & 0.464 & 5.364 \\
\hline
\end{tabular}

Table 6. Paired $t$-Student criterion with one sided distribution $\left(t_{\exp }\right)$, women at the age of $20-22$

\begin{tabular}{||l|c|c|c|c||}
\hline \multicolumn{1}{|c|}{ Parameters } & $S[\mathrm{mmHg}]$ & $D[\mathrm{mmHg}]$ & $\varphi[D / S]$ & $H R[$ beats/min] \\
\hline \hline$E_{\max }[\mathrm{V} / \mathrm{m}]$ & 2.218 & 2.979 & 2.634 & 1.182 \\
\hline$H_{\max }[\mathrm{mA} / \mathrm{m}]$ & 5.483 & 7.237 & 3.926 & 2.292 \\
\hline$\mu_{\max }\left[\mathrm{mW} / \mathrm{cm}^{2}\right]$ & 2.176 & 4.343 & 0.001 & 1.447 \\
\hline$E_{\text {aver }}[\mathrm{V} / \mathrm{m}]$ & 3.401 & 2.907 & 7.574 & 2.540 \\
\hline$H_{\text {aver }}[\mathrm{mA} / \mathrm{m}]$ & 1.299 & 1.267 & 2.977 & 2.175 \\
\hline$\mu_{\text {aver }}\left[\mathrm{mW} / \mathrm{cm}^{2}\right]$ & 2.550 & 3.896 & 0.349 & 1.982 \\
\hline
\end{tabular}

The number of degrees of freedom $f$ for all calculations is as follows $(122+122)-$ $-2=242$. In the table we find the value Student $t$-test (at $p=0.05)-1.960\left(t_{\text {tabl }}\right)$.

The positive (significant) index of correlation is when $\left|t_{\exp }\right|>t_{\text {tabl }}$. 


\section{Conclusions}

The conducted research and calculations suggest that the value of the human arterial pressure and heart rate in general correlate with indicators of the electromagnetic field of the urban social ecological system in Ivano-Frankivsk city. In particular:

- There is a statistically significant negative index correlation between the values of heart rate [beats/min.] and $E_{\text {aver }}[\mathrm{V} / \mathrm{m}], H_{\text {aver }}[\mathrm{mA} / \mathrm{m}], \mu_{\text {aver }}\left[\mathrm{mW} / \mathrm{cm}^{2}\right]$ for men and women at the age of 20-22 showing an inverse relationship between these values at moderate correlation ratio between them.

- There is a statistically significant negative index of correlation between arterial pressure $\varphi$ and $E_{\max }[\mathrm{V} / \mathrm{m}], H_{\max }[\mathrm{mA} / \mathrm{m}]$ for men and women the age of 20-22 showing an inverse relationship between these values at moderate correlation ratio between them.

- The moderate correlation ratio and negative index of correlation in men at the age of 20-22 and the lower correlation ratio and negative index of correlation in women at the age of 20-22 are statistically significant for indicators of diastolic arterial pressure $D[\mathrm{mmHg}]$ and $E_{\max }[\mathrm{V} / \mathrm{m}]$.

- For the parameters of systolic arterial pressure $S[\mathrm{mmHg}]$ and electromagnetic field indicators of urban social ecological system in Ivano-Frankivsk city significant correlation relationships were not found.

\section{References}

[1] Bezverkhaya A.P.: Gigiyenicheskaya otsenka vliyaniya elektromagnitnogo izlucheniya na organizm cheloveka $i$ zhivotnykh [Гигиеническая оценка вдияния электромагнитного излучения на организм человека и животных]. Hihiyena naselenykh mists', Kyyiv 2009.

[2] Burlaka N.I., Gozhenko S.S.: Elektromagnitnoye pole, yego vidy, kharakteristiki, klassifikatsiya i vliyaniye na zdorov'ye naseleniya [Электромагнитное поле, его виды, характеристики, классификация и влияние на здоровье населения]. Aktual'ni problemy transportnoyi medytsyny, nr 4 (2), 2010, pp. 24-32.

[3] Vadzyuk S.N., Denefil' O.V.: Osoblyvosti psykhofiziolohichnoho stanu osib cholovichoyi ta zhinochoyi stati za riznykh typiv pohody [Особливості психофізіологічного стану осіб чоловічої та жіночої статі за різних типів погоди]. Hihiyena naselenykh mists', Kyyiv 2004.

[4] Hrachëv N.N.: Mediko-biologicheskiye aspekty vozdeystviya EMI [Медико-биологические аспекты воздействия ЭМИ]. [on-line:] http://grachev.distudy. ru/Uch_kurs/sredstva/Templ_1/templ_1_4.html [access: 15.04.2015]. 
[5] Denefil' O.V.: Pokaznyky kardiohemodynamiky u studentok z riznoyu masoyu tila za medyko-meteorolohichnoyi sytuatsiyi I, II i III typiv [Показники кардіогемодинаміки у студенток з різною масою тіла за медико-метеорологічної ситуації I, II і III типів]. Arkhiv klinichnoyi medytsyny, Kyyiv 2008.

[6] Nikitina N.H.: Vplyv elektromahnitnykh vyprominyuvan' na zdorov"ya naselennya (naukovyy ohlyad) [Вплив електромагнітних випромінювань на здоров' я населення (науковий огдяд)]. Hihiyena naselenykh mists', Kyyiv 2007.

[7] Merdukh I.I., Labiy Yu.M., Krupchuk T.Yu.: Sposib otsinky oplyou seredovyshcha na sertsevo-sudynnu systemu lyudyny [Спосіб оцінки впливу середовища на серцево-судинну систему людини]. Patent 76173 Ukrayina 2012.

[8] Selyuk M.M.: Vplyv elektromahnitnykh poliv nadoysokoho diapazonu na sertsevo-sudynnu systemи [Вплив електромагнітних полів надвисокого діапазону на серцево-судинну систему]. [on-line:] http://www.mif-ua.com/ archive/article/10683 [access:15.04.2015].

[9] Tarasova V.V.: Ekolohichna statystyka (z blochno-modul'noyu formoyu kontrolyu znan') [Екологічна статистика (з блочно-модульною формою контролю знань)]. Tsentr uchbovoyi literatury, Kyyiv 2008.

[10] Shevchuk V.H.: Fiziolohiya [Фізіологія]. Nova Knyha, Vinnytsya 2012.

[11] http://www.medstatistic.ru/theory/t_cryteria.html.

[12] http://www.mvk.if.ua/aboutcity.

[13] http://www.tenmars.com/webls-en-us/TM-195.html. 